\title{
MicroRNA-125a-5p enhances the sensitivity of esophageal squamous cell carcinoma cells to cisplatin by suppressing the activation of the STAT3 signaling pathway
}

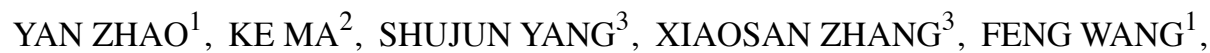 \\ XIAQING ZHANG ${ }^{4}$, HONGTAO LIU $^{4}$ and QINGXIA FAN ${ }^{1}$
}

\author{
${ }^{1}$ Department of Oncology, The First Affiliated Hospital of Zhengzhou University, Zhengzhou, Henan 450052; \\ ${ }^{2}$ College of Traditional Chinese Medicine, Shandong University of Traditional Chinese Medicine, Jinan, Shandong 250355; \\ ${ }^{3}$ Department of Oncology, The Affiliated Cancer Hospital of Zhengzhou University, Zhengzhou, Henan 450003; \\ ${ }^{4}$ College of Life Sciences, Zhengzhou University, Zhengzhou, Henan 450001, P.R. China
}

Received February 12, 2018; Accepted May 9, 2018

DOI: 10.3892/ijo.2018.4409

\begin{abstract}
Increasing evidence has demonstrated that microRNAs (miRNAs or miRs) play a variety of roles in tumor development, progression and chemosensitivity in a wide range of tumors. In this study, we found that miR-125a-5p exhibited a low expression in esophageal squamous cell carcinoma (ESCC) tissues and cells, and that its low expression was associated with higher tumor staging and shorter a survival time of patients with ESCC. Moreover, miR-125a-5p overexpression contributed to the suppression of cell proliferation, cell cycle arrest, cell apoptosis and a decrease in cell migratory and invasive abilities, whereas the downregulation of miR-125a-5p promoted cell proliferation, accelerated cell cycle progression, suppressed apoptosis and enhanced the migratory and invasive abilities of ESCC EC1 and TE1 cells, which may be tightly associated with the epithelial-mesenchymal transition (EMT) process in ESCC. Importantly, miR-125a-5p enhanced the cytotoxic effects of cisplatin on EC1 and TE1 cells, and co-treatment with miR-125a-5p and cisplatin significantly induced cell apoptosis and reduced the cell migratory and invasive abilities of EC1 and TE1 cells, coupled with an increase in the E-cadherin level and a decrease in the $\mathrm{N}$-cadherin and Vimentin levels. Most notably, signal transducer and activator of transcription-3 (STAT3) was found to be a direct target of miR-125a-5p in ESCC cells, and miR-125a-5p overexpression significantly reduced the protein levels of t-STAT3, p-STAT3 and vascular endothelial growth factor (VEGF) in EC1 and TE1 cells. Furthermore, the combination of miR-125a-5p and
\end{abstract}

Correspondence to: Professor Qingxia Fan, Department of Oncology, The First Affiliated Hospital of Zhengzhou University, 1 East Jianshe Road, Zhengzhou, Henan 450052, P.R. China E-mail: fanqingxia2011@126.com

Key words: esophageal squamous cell carcinoma, epithelialmesenchymal transition, microRNA-125a-5p, cisplatin, cytotoxicity, signal transducer and activator of transcription-3 cisplatin markedly inactivated the STAT3 signaling pathway; however, interleukin (IL)-6, a widely reported activator of the STAT3 signaling pathway, reversed the suppressive effects of miR-125a-5p/cisplatin in ESCC cells on the activation of the STAT3 signaling pathway. Of note, we found that IL-6 markedly reversed the altered cell phenotype mediated by the combination of miR-125a-5p and cisplatin in ESCC cells. These findings suggest that miR-125a-5p may play a pivotal role in the development and progression of ESCC, which may be achieved via the manipulation of the STAT3 signaling pathway.

\section{Introduction}

Esophageal carcinoma (EC) is one of the most frequently occurring malignances worldwide (1). EC is divided into two main histological types, including esophageal squamous cell carcinoma (ESCC) and esophageal adenocarcinoma (ECA); ESCC as a main histological type is more common in Africa, Iran and North China $(2,3)$. Despite tremendous advances being made in therapeutic strategies, the 5-year survival rate for patients with ESCC remains markedly poor (4-8). Notably, even though chemotherapy is an effective therapeutic approach for patients with ESCC (9), the development of drug resistance has become the most severe concern, and is the main cause of treatment failure in patients with ESCC (10). Therefore, it is imperative to elucidate the mechanisms responsible for drug resistance in order to improve the survival rate of patients with ESCC.

MicroRNAs (miRNAs or miRs) are a class of small non-coding RNAs comprising of 19-25 nucleotides in length, which regulate geneexpression by targeting related genes $(11,12)$. Ample evidence has revealed that miRNAs are involved in a number of cellular processes, such as cell apoptosis, cell cycle, cell invasion and metastasis, the regulation of signaling networks and drug resistance (13-19). Furthermore, miRNAs are tightly associated with tumor initiation, development and progression in a variety of tumors via the modulation of their target gene levels (20-23); hence, miRNAs may function as 
either oncogenes or tumor suppressors in different types of tumors (20,24-27). Therefore, it is imperative to interpret the function of miRNAs in the occurrence and development of a large number of tumors, which suggests that miRNAs, as novel and promising therapeutic targets, may exhibit huge clinical value in the future. In addition, miRNAs may function as molecular markers for early the diagnosis of a number of tumors $(28,29)$, which may aid in the development of a number of diagnostic agents for multiple tumor types. miRNA-125a-5p (miR-125a-5p), a type of newly discovered miRNA molecule, has been verified to be involved in the development and progression of a number of tumors, including laryngeal cancer (30), hepatocellular carcinoma (31-33), lung cancer (34) and prostate carcinoma (35). The tumor suppressive function of miR-125a-5p has also been supported by a number of investigations on a variety of tumor types $(32,36,37)$. Notably, miR-125a has been shown to enhance the sensitivity of paclitaxel-resistant colon cancer cells to paclitaxel (38), suggesting that miR-125a may prove to be a novel ancillary drug for use in chemotherapy for patients with tumors. These data imply that miR-125a-5p has tremendous potential for use in the diagnosis, treatment and prognosis of a wide range of tumors.

In the current study, we examined miRNA-125a-5p expression in ESCC tissues and cell lines, and verified its role in the regulation of the proliferation, the cell cycle, apoptosis, and in the migratory and invasive abilities of ESCC cells. miR-125a-5p was found to enhance the sensitivity of ESCC cells to cisplatin by suppressing the activation of the signal transducer and activator of transcription-3 (STAT3) signaling pathway. Most importantly, interleukin (IL)-6, a widely reported activator of the STAT3 signaling pathway (39), abrogated the inactivated status of the STAT3 signaling pathway elicited by the combined use of miR-125a-5p and cisplatin, which was accompanied by cell phenotypic recovery. Taken together, the data from the current study suggest that the manipulation of miR-125a-5p may be used as a strategy with which to enhance the cytotoxic effects of cisplatin on ESCC via the suppression of the activation of the STAT3 signaling pathway.

\section{Materials and methods}

Patients and tissue samples. This study was approved by the Institutional Research Ethics Committee of Zhengzhou University. A total of 56 cases of ESCC tissues and paired normal esophageal epithelial tissues were treated with surgical resection alone from the First Affiliated Hospital of Zhengzhou University, Zhengzhou, China from May, 2010 to August, 2012. The clinical characteristics of the patients with ESCC are summarized in Table I. All tissue samples were confirmed by a pathologist. All samples were obtained with informal written and none of the patients had received any treatments prior to surgery. The tissues were immediately frozen in liquid nitrogen until RNA extraction.

Cell lines and cell culture. The human ESCC cell lines, including Eca109, EC9706, EC1, TE1, KYSE450 and KYSE70, as well as normal esophageal epithelial cells, Het-1A, were maintained in liquid nitrogen in our laboratory. The cell lines above were cultured in RPMI-1640 medium supplemented with $10 \%$ fetal bovine serum (FBS; Gibco/Thermo Fisher Scientific, Grand Island, NY, USA), $100 \mathrm{U} / \mathrm{ml}$ penicillin and $100 \mu \mathrm{g} / \mathrm{ml}$ streptomycin (both from Sigma-Aldrich, St. Louis, $\mathrm{MO}, \mathrm{USA}$ ) in a humidified $5 \% \mathrm{CO}_{2}$ incubator at $37^{\circ} \mathrm{C}$.

Cell transfection. The miR-125a-5p mimic, miR-125a-5p inhibitor and the negative control (NC) (Ribobio, Guangzhou, China) at a final concentration of $30 \mathrm{nM}$ were transfected into the EC1 and TE1 cells using Lipofectamine ${ }^{\mathrm{TM}} 2000$ (Invitrogen/Life Technologies, Carlsbad, CA, USA) according to manufacturer's instructions. All transfection experiments were performed in triplicate for each treatment group at 24, 48, 72 and $96 \mathrm{~h}$ for cell proliferation assay and at $48 \mathrm{~h}$ for the other experiments.

Prediction of target genes. The potential target genes of miR-125a-5p were searched using online webpage TargetScan (http://www.targetscan.org/vert_72/), miRanda (http://www. microrna.org/microrna/home.do) and miRDB (http://www. mirdb.org/).

Plasmid construction and luciferase reporter assay. The human STAT3 3'-UTR-wild-type (STAT3-3'-UTR-WT) region containing the miR-125a-5p binding sequence was amplified by PCR, and the STAT3-3'-UTR-mutation (STAT3-3'-UTR-MUT) region with a substitution of $8 \mathrm{bp}$ in the miR-125a-5p binding region was generated using a QuikChange Site-Directed Mutagenesis kit (Stratagene, La Jolla, CA, USA). The STAT3-3'-UTR-WT and STAT3-3'-UTR-MUT were inserted into the downstream region of the Firefly luciferase gene, respectively. The EC1 and TE1 cells were co-transfected using reporter plasmids (400 per $20 \mathrm{ng}$ internal control Renilla luciferase plasmid pRL-SV40) and miR-125a-5p mimic or NC by Lipofectamine 2000 (Invitrogen/Life Technologies) according to the manufacturer's instructions. Subsequnetly, luciferase activity was determined using the Dual Luciferase Assay kit (Promega, Madison, WI, USA) using a Synergy H1 hybrid reader (Biotek, Winooski, VT, USA) at $48 \mathrm{~h}$ following transfection. Finally, the luciferase activity was normalized to the Renilla luciferase activity.

Reverse transcription-quantitative PCR (RT-qPCR). Total RNA was isolated from the tissues and cells, and subjected to miRNA First Strand cDNA Synthesis kit (cat. no. B532453; Sangon Biotech, Shanghai, China) using the specific miR-125a-5p reverse transcription primer, 5'-CTCAACTGGTGTCGTGGAGTCGG CAATTCAGTTGAGTCACAGGT-3' and the U6 gene reverse transcription primer, 5'-GTCGTATCCAGTGCAGGGTCCG AGGTATTCGCACTGGATACGACAAAATA-3'. Quantitative PCR (qPCR; Tiangen Biotech, Beijing, China) was used to determine miR-125a-5p expression using the ABI 7500 Real-time PCR System (Applied Biosystems, Foster City, CA, USA) by the addition of miR-125a-5p specific amplification primers as follows: 5'-ACACTCCAGCTGGGTCCCTGAGACCCTTT AAC-3' (forward) and 5'-TGGTGTCGTGGAGTCG-3' (reverse).

Western blot analysis. Total proteins were isolated from the ESCC cells using RIPA lysis buffer (Solarbio, Beijing, China). The protein concentration was determined using a Micro BCA 
Protein Assay kit (cat. no. 23235; Pierce Biotechnology, Inc., Rockford, IL, USA). The proteins (50 $\mu \mathrm{g} /$ lane) were separated by $12 \%$ SDS-PAGE, and then transferred onto PVDF membranes (Millipore Corporation, Billerica, MA, USA). After blocking with skimmed milk, primary antibodies against E-cadherin (cat. no. ab1416, 1:50 dilution), N-cadherin (cat. no. ab98952, 1:500 dilution), Vimentin (cat. no. ab8978, 1:100 dilution), VEGF (cat. no. ab69479, 1:100 dilution), $\beta$-actin (cat. no. ab8226, 1:500 dilution) (all from Abcam, Cambridge, MA, USA), total STAT3 (t-STAT3, cat. no. 9139, 1:1,000 dilution) and phosphorylated STAT3 (p-STAT3, cat. no. 4113, 1:1,000 dilution) (all from Cell Signaling Technology, Beverly, MA, USA) were incubated with the PVDF membranes (Millipore Corporation) overnight at room temperature. Subsequently, Horseradish peroxidase (HRP)-labeled secondary antibody (cat. no. SE131, 1:5,000 dilution; Solarbio) was applied to the PVDF membranes. Finally, the protein signal was developed using enhanced chemiluminescence reagents (Beyotime Biotech, Haimen, China). The densitometry of the protein bands was performed using Image J software v1.8.0 (National Institutes of Health, Bethesda, MD, USA).

Cell counting kit-8 (CCK-8) assay. The EC1 and TE1 cells $(2,000$ cells/well) were seeded into a 96 -well plate, and these cells were then transfected with miR-125a-5p mimic, miR-125a-5p inhibitor and NC, and treated with cisplatin $(0$, 1, 2, 5, 10 and $15 \mu \mathrm{g} / \mathrm{ml}$; Hansoh Pharmaceutical Co. Ltd., Jiangsu, China) or IL-6 (20 $\mu \mathrm{g} / \mathrm{ml}$; PeproTech Inc., Rocky Hill, NJ, USA) in triplicate were applied to the corresponding wells. Cell viability was determined using the CCK-8 kit (Beyotime Biotech) according to the manufacturer's instructions by measuring the absorbance at $450 \mathrm{~nm}$ on a microplate reader (Thermo Fisher Scientific, Waltham, MA, USA).

Cell cycle detection. Cell cycle assay was conducted as described in a previous study (40). Briefly, the EC1 and TE1 cells were harvested at $48 \mathrm{~h}$ following transfection with miR-125a-5p mimic, miR-125a-5p inhibitor or NC, rinsed using PBS and fixed in $70 \%$ ethanol overnight at $4^{\circ} \mathrm{C}$. After rinsing thrice, propidium iodide (PI; Sigma-Aldrich) was used to treat the cells, and a flow cytometer (BD Biosciences, San Diego, CA, USA) was used to detect the DNA contents.

Cell apoptosis assay. Cell apoptosis assay was performed as described in a previous study (40) using Annexin V FITC/PI (Sigma-Aldrich). In brief, the EC1 and TE1 cells were collected using trypsinase, and Annexin V/PI reagents were added to the cells for $30 \mathrm{~min}$. Finally, a flow cytometer (BD Biosciences) was utilized to determine cell apoptosis.

Would healing assay. Cell migration was investigated by wound healing migration assay as previously described (41). Briefly, a Ibidi Culture-Insert 2 well (Ibidi Company, Martinsried, Germany) was placed in a 24-well plate, and the EC1 and TE1 cells transfected with miR-125a-5p mimic, miR-125a-5p inhibitor or NC were digested and seeded into 24-well culture plates at a density of $5 \times 10^{5}$ cells/well using RPMI-1640 medium containing 10\% FBS. The Culture-Insert 2 well was gently removed $24 \mathrm{~h}$ following appropriate cell attachment. At 0,12 and $24 \mathrm{~h}$, images were obtained at the same position
Table I. Clinical characteristics of the patients with esophageal squamous cell carcinoma.

\begin{tabular}{lc}
\hline Characteristic & $\mathrm{n}=56$ \\
\hline Age (years) & \\
$\geq 60$ & 33 \\
$<60$ & 23 \\
Sex & \\
Male & 38 \\
Female & 18 \\
TNM staging & \\
I and II & 26 \\
III and IV & 30 \\
Histological grade & \\
High differentiation & 15 \\
Moderate differentiation & 19 \\
Poor differentiation & 22 \\
Lymph node metastasis & \\
Yes & 21 \\
No & 35 \\
\hline
\end{tabular}

under an inverted microscope (Nikon Instruments, Tokyo, Japan), respectively. The migration distances were quantified by measuring the distances from the wound edges.

Cell invasion assay. Cell invasion assay was performed using a Transwell chamber with Matrigel (BD Biosciences). Briefly, the EC1 and TE1 cells at a density of $1 \times 10^{5}$ were added to the upper layer of the chamber, and 20\% FBS was added to the bottom layer of the chamber. Invasive cells were fixed using methanol and stained with crystal violet (Sigma-Aldrich) for $5 \mathrm{~min}$ at room temperature $48 \mathrm{~h}$ after treatment. Finally, invasive cell numbers were counted under a field of x 200 magnification under an inverted fluorescence microscope (Nikon Instruments).

Statistical analysis. Statistical analysis was performed using GraphPad Prism 6.0 software. Data are expressed as the means $\pm \mathrm{SD}$, derived from experiments with at least 3 independently repeats. Comparisons between two groups were made using the Student's t-test, and comparisons between more than two were made using one-way ANOVA followed by Dunnett's test. Values of $\mathrm{P}<0.05$ were considered to indicate statistically significant differences.

\section{Results}

Decreased expression of miR-125a-5p in ESCC tissues and cells. Ample evidence has demonstrated that miRNAs are widely involved in tumor development and progression $(42,43)$. In this study, in order to determine whether miR-125a-5p is tightly associated with the occurrence and development of ESCC, we examined its expression patterns in ESCC tissues and cells. We found that relative level of miR-125a-5p in ESCC tissues was significantly lower than that in normal 
A

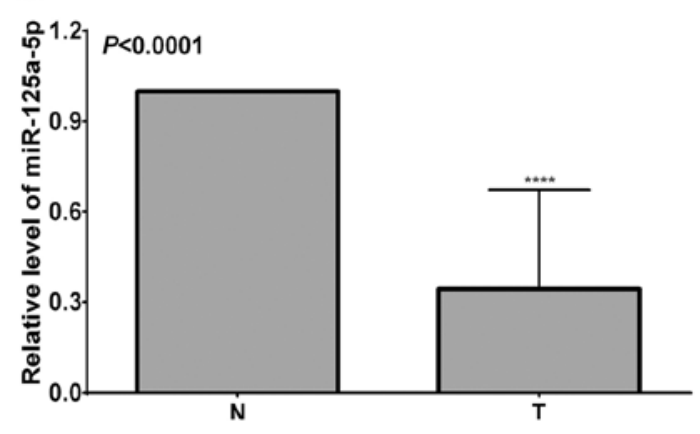

C

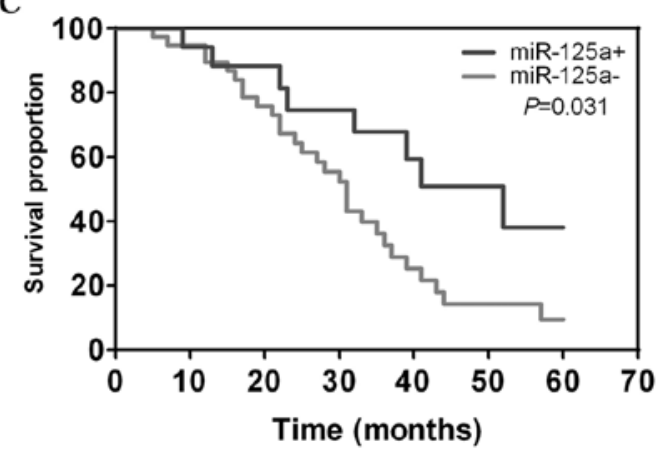

B
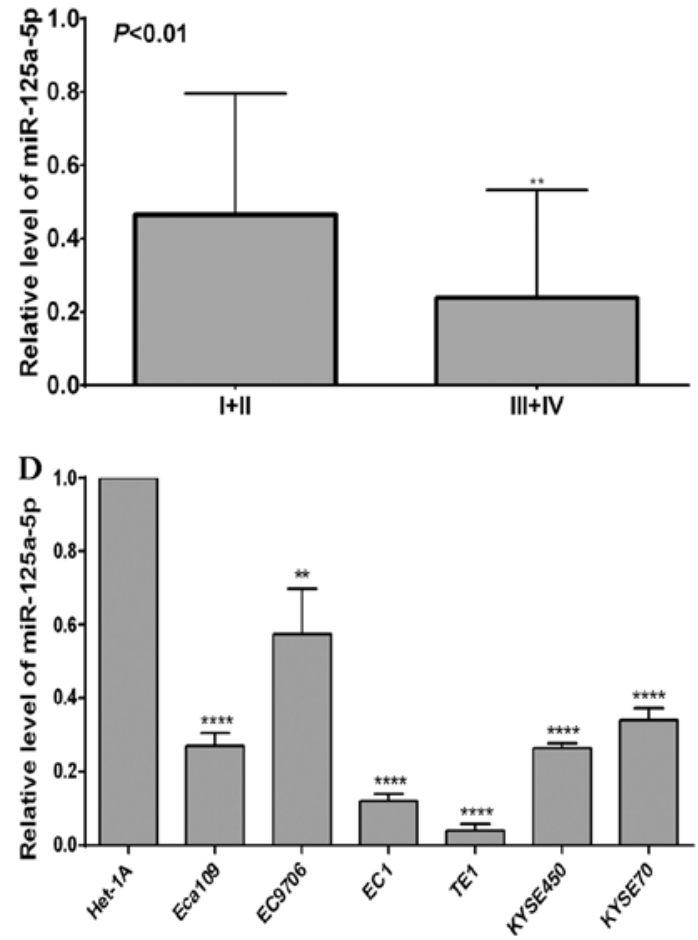

Figure 1. Expression pattern of miR-125a-5p in esophageal squamous cell carcinoma (ESCC) and its association with the prognosis of patients with ESCC. (A) miR-125a-5p level in normal esophageal epithelial tissues (N) and ESCC tissues (T). Total RNA was isolated from 56 ESCC tisues and paired normal esophageal epithelial tissues, and subjected to analysis using the cDNA synthesis kit. RT-qPCR was used to detect the miR-125a-5p level in ESCC tissues and paired normal esophageal epithelial tissues; ${ }^{* * * *} \mathrm{P}<0.0001$, compared with normal tissues. (B) Expression of miR-125a-5p is tightly associated with tumor TNM staging. TNM staging in a variety of ESCC tissues was confirmed by pathology, and RT-qPCR was used to detect the miR-125a-5p level in ESCC tissues; ${ }^{* *} \mathrm{P}<0.01$, compared with ESCC with I and II staging. (C) Association of miR-125a-5p level with the prognosis of the patients with ESCC. Kaplan-Meier analysis was used to evaluate the association of miR-125a-5p with the overall survival rate, and the log-rank (Mantel-Cox) test was used to determine the difference between positive miR-125a-5p and negative miR-125a-5p expression. (D) Expression of miR-125a-5p in various ESCC cell lines (Eca109, EC9706, EC1, TE1, KYSE450 and KYSE70 cells) and the normal esophageal epithelial cell line, Het-1A; ${ }^{* *} \mathrm{P}<0.01$ and ${ }^{* * * *} \mathrm{P}<0.0001$, compared with the Het-1A cells.

esophageal epithelial tissues $(\mathrm{P}<0.0001)$ (Fig. 1A). Further analysis revealed that the relative level of miR-125a-5p in the patients with I + II stage disease was markedly higher than in those with III + IV stage disease $(\mathrm{P}<0.01)$ (Fig. 1B), suggesting that miR-125a-5p is tightly associated with the tumor clinical staging in ESCC. To further determine the prognostic value of miR-125a-5p, Kaplan-Meier survival analysis was used to evaluate the association of the miR-125a-5p expression level with the prognosis of patients with ESCC. The results revealed that a high expression of miR-125a-5p contributed to a better overall survival of patients with ESCC (Fig. 1C). Furthermore, an in vitro analysis demonstrated that the relative level of miR-125a-5p in ESCC cells (Eca109, EC9706, EC1, TE1, KYSE450 and KYSE70) was evidently lower than that in the normal esophageal epithelial cell line, Het-1A $(\mathrm{P}<0.01)$ (Fig. 1D), which further supported the data obtained from ESCC tissues. These findings suggest that miR-125a-5p is involved in the development, progression and prognosis of ESCC and that its upregulation contributes to an improved prognosis of patients with ESCC. Therefore, it is very imperative to examine the function of $\mathrm{miR}-125 \mathrm{a}-5 \mathrm{p}$ in the occurrence and development of ESCC.

Role of miR-125a-5p in the regulation of the proliferation, cell cycle and apoptosis of ESCC cells. To preliminarily elucidate the underlying function of miR-125a-5p in ESCC, we transfected
NC, miR-125a-5p mimic and miR-125a-5p inhibitor into EC1 and TE1 ESCC cells, and RT-qPCR was employed to examine miR-125a-5p expression in the ESCC cells. We found that relative level of miR-125a-5p in the miR-125a-5p mimic group was significantly higher than that in the control group and NC group in the EC1 and TE1 cells $(\mathrm{P}<0.05)$, whereas transfection with the miR-125a-5p yielded opposite results in the EC1 and TE1 cells (Fig. 2A). Further analysis using CCK-8 assay confirmed that miR-125a-5p overexpression contributed to the suppression of the proliferation of the EC1 and TE1 cells at various time points, including 24, 48, 72 and $96 \mathrm{~h}$, compared with the control group and $\mathrm{NC}$ group $(\mathrm{P}<0.05)$, and opposite data were observed in the miR-125a-5p inhibitor group (Fig. 2B). Cell cycle analysis revealed that the cell numbers in the G0/G1 phase in the miR-125a-5p mimic group were markedly higher than those in the control group and NC group and opposite results were observed with the cells in the $\mathrm{S}$ phase $(\mathrm{P}<0.05)$; however, transfection with miR-125a-5p inhibitor markedly decreased the cell numbers in the G0/G1 phase and increased the cell numbers in the $\mathrm{S}$ phase compared with the control group and NC group (Fig. 2C and D). Moreover, cell apoptosis assay revealed that miR-125a-5p upregulation markedly promoted the apoptosis of the EC1 and TE1 cells, and miR-125a-5p downregulation evidently suppressed cell apoptosis, compared with the control group and NC group (Fig. 2E and F). These 


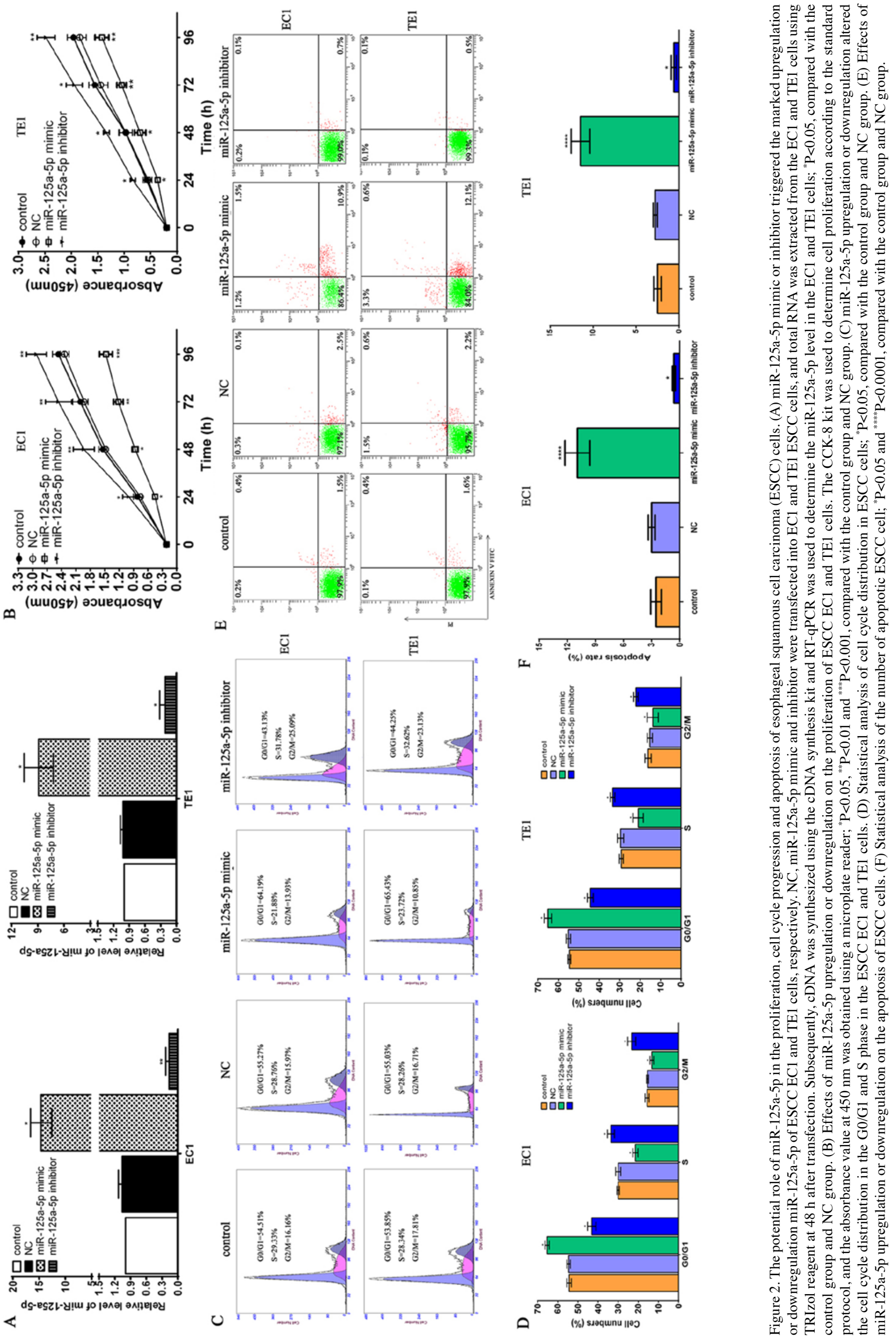


data suggest that miR-125a-5p functions as a tumor suppressor in ESCC.

miR-125a-5p suppresses the migratory and invasive abilities of ESCC cells via the regulation of the epithelial-mesenchymal transition (EMT) process. Accumulating evidence has demonstrated that miRNAs are widely involved in the migration and invasion in a wide range of tumors $(44,45)$. However, whether miR-125a-5p is tightly associated with the invasion and metastasis of ESCC remains undetermined. Thus, in this study, we examined the effects of miR-125a-5p mimic or inhibitor on the migratory and invasive abilities of EC1 and TE1 cells. The results from wound healing assay and Transwell chamber assay revealed that miR-125a-5p overexpression significantly suppressed the cell migratory and invasive abilities; conversely, miR-125a-5p downregulation markedly promoted the migratory and invasive abilities of the EC1 and TE1 cells, compared with the control group and NC group $(\mathrm{P}<0.01)$ (Fig. 3A-D). A number of studies have revealed that EMT plays an important role in invasion, metastasis, and carcinogenesis in a variety of tumors $(46,47)$. Thus, in this study, to elucidate the underlying mechanisms responsible for the suppressive effects of miR-125a-5p on the invasive ability of ESCC cells, we detected the expression levels of EMT-related molecular markers in ESCC cells. We found that miR-125a-5p overexpression led to E-cadherin upregulation, and the downregulation of $\mathrm{N}$-cadherin and Vimentin in the EC1 and TE1 cells, whereas miR-125a-5p downregulation decreased the E-cadherin level, and enhanced the levels of $\mathrm{N}$-cadherin and Vimentin, compared with the control group and NC group (Fig. 3E and F), suggesting miR-125a-5p inhibits the EMT process in ESCC. These findings indicate that miR-125a-5p mediates the suppression of the migration and invasion of ESCC cells via the inhibition of the EMT process.

miR-125a-5p upregulation enhances the cytotoxic effects of cisplatin, induces cell apoptosis and reduces the migratory and invasive abilities of ESCC cells. Chemoresistance is the main cause of tumor treatment failure, and aberrant miRNA levels are closely linked to chemosensitivity and chemoresistance in a wide range of tumors. To further investigate whether miR-125a-5p is tightly implicated in chemosensitivity and chemoresistance in ESCC cells, in this study, we investigated the effects of various concentrations of cisplatin combined with NC or miR-125a-5p transfection on the proliferation, apoptosis, migration and invasion of ESCC cells. We found that miR-125a-5p significantly enhanced the cytotoxic effects of cisplatin on EC1 and TE1 cells, compared with NC group treated with cisplatin (Fig. 4A). Further analysis revealed that transfection with miR-125a-5p mimic alone or treatment with cisplatin alone markedly induced cell apoptosis and reduced the migratory and invasive abilities of the EC1 and TE1 cells, compared with the NC group $(\mathrm{P}<0.05)$ (Fig. 4B-G). However, miR-125a-5p in combination with cisplatin was the most effective in the induction of cell apoptosis and the decrease in the migratory and invasive abilities of the EC1 and TE1 cells (Fig. 4B-G). To further elucidate the underlying mechanisms of the combined effects of miR-125a-5p and cisplatin, we further examined the levels of the EMT-related proteins,
E-cadherin, N-cadherin and Vimentin. We found that transfection with miR-125a-5p alone, and treatment with cisplatin alone or their combination significantly promoted E-cadherin expression, and suppressed the expression levels of $\mathrm{N}$-cadherin and Vimentin, compared with the NC group $(\mathrm{P}<0.05)$ in the EC1 and TE1 cells. However, the combined use of miR-125a-5p and cisplatin was the most effective in suppressing the EMT process (Fig. 4H and I). These data indicate that miR-125a-5p plays a pivotal role in enhancing the cisplatin-mediated chemosensitivity of ESCC cells via suppressing the EMT process.

STAT3 is a direct target of miR-125a-5p in ESCC. To clarify the possible molecular mechanisms of the chemosensitivity triggered by miR-125a-5p in ESCC, we performed a search for the potential target genes of miR-125a-5p using TargetScan, miRanda and miRDB. We found that STAT3 was a potential target gene of miR-125a-5p (Fig. 5A), and corresponding STAT3-3'-UTR-WT and STAT3-3'-UTR-MUT plasmids were constructed (Fig. 5A). Subsequently, these vectors, along with NC or miR-125a-5p were co-transfected into the EC1 and TE1 cells, and the luciferase activity was determined by measuring the relative luciferase intensity. We found that miR-125a-5p significantly decreased the luciferase activity in the STAT3-3'UTR-WT group, but it did not affect the luciferase activity in the cells in the STAT3-3'UTR-MUT group (Fig. 5B and C), suggesting that miR-125a-5p can directly bind to the 3'UTR region of STAT3. To validate the results mentioned above, we further performed western blot analysis to detect the expression levels of t-STAT3, p-STAT3 and its downstream target gene, VEGF, in the EC1 and TE1 cells. The results demonstrated that miR-125a-5p overexpression significantly decreased the protein levels of t-STAT3, p-STAT3 and VEGF in the EC1 and TE1 cells, compared with the control and NC group ( $\mathrm{P}<0.01)$ (Fig. 5D-G). These findings suggest that STAT3 is a direct target gene of miR-125a-5p, and that miR-125a-5p suppresses the invasive ability of ESCC cells, and enhances chemosensitivity and that these effects may be mediated via the suppression of the activation of the STAT3 signaling pathway in ESCC.

Combination of miR-125a-5p with cisplatin inactivates the STAT3 signaling pathway in ESCC cells. To further explore the underlying mechanisms of the antitumor effects mediated by miR-125a-5p in combination with cisplatin in ESCC, we examined the protein expression levels of t-STAT3, p-STAT3 and VEGF in ESCC cells subjected to different treatments. We found that $\mathrm{miR}-125 \mathrm{a}-5 \mathrm{p}$ alone or cisplatin alone significantly downregulated the protein levels of t-STAT3, p-STAT3 and VEGF, compared with the NC group ( $\mathrm{P}<0.01)$ (Fig. 6A and $\mathrm{B}$ ). However, the combination of miR-125a-5p with cisplatin exerted the most prominent inhibitory effect on the expression levels of these proteins (Fig. 6A and B). These findings suggest that miR-125a-5p and cisplatin play a synergistic antitumor role in ESCC via suppressing the activation of the STAT3 signaling pathway.

IL-6 attenuates the inhibitory effects of miR-125a-5p combined with cisplatin on the activation of the STAT3 signaling pathway in ESCC cells. To determine whether the re-activation of the STAT3 signaling pathway evoked by IL-6 (a widely 

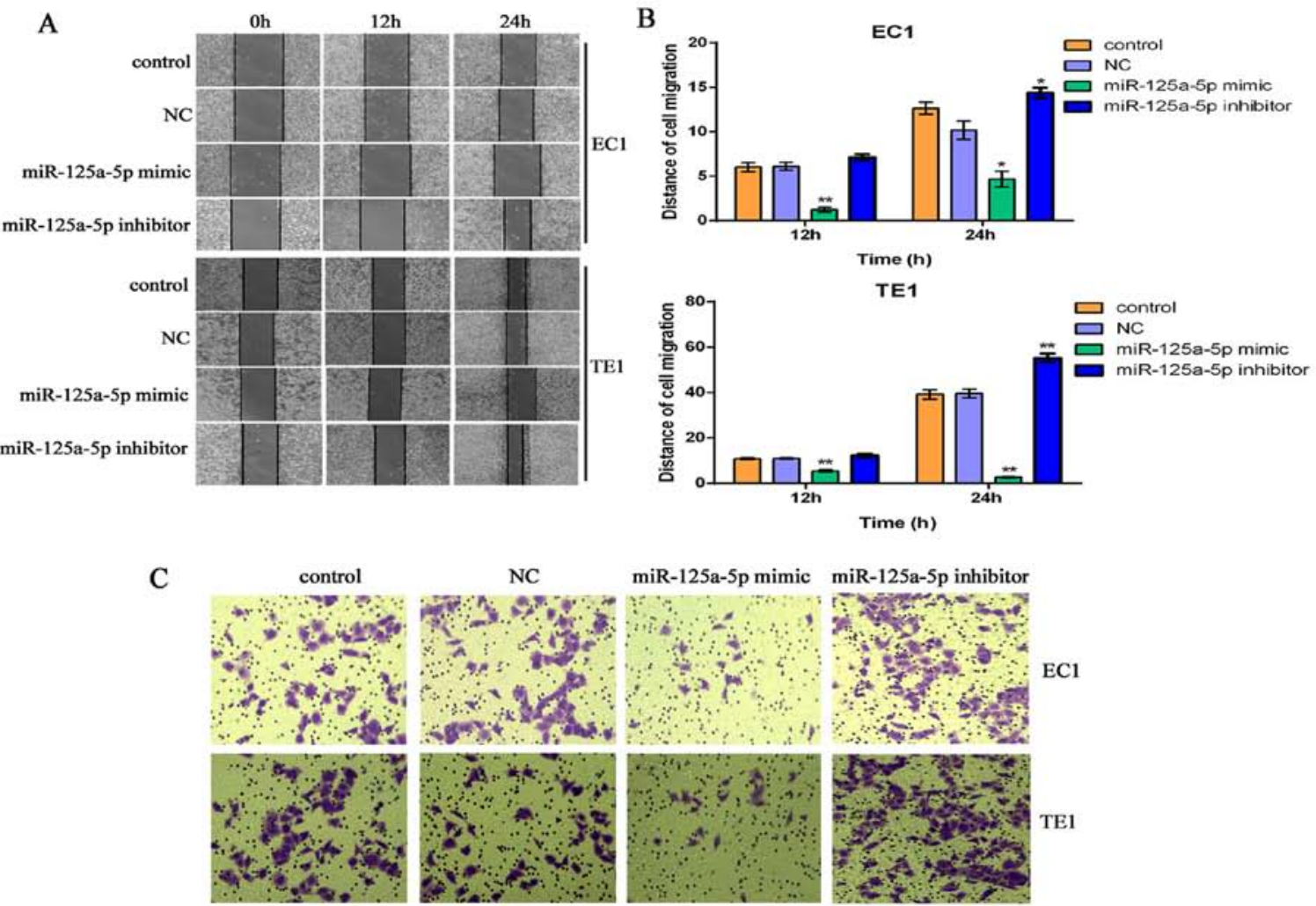

miR-125a-5p mimic miR-125a-5p inhibitor

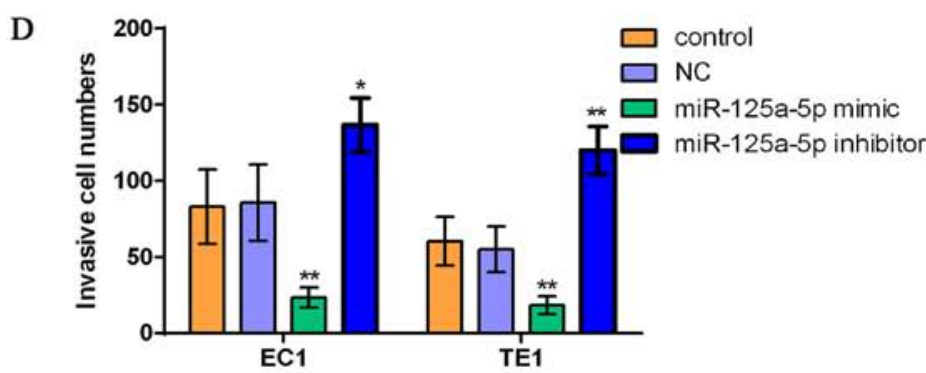

E
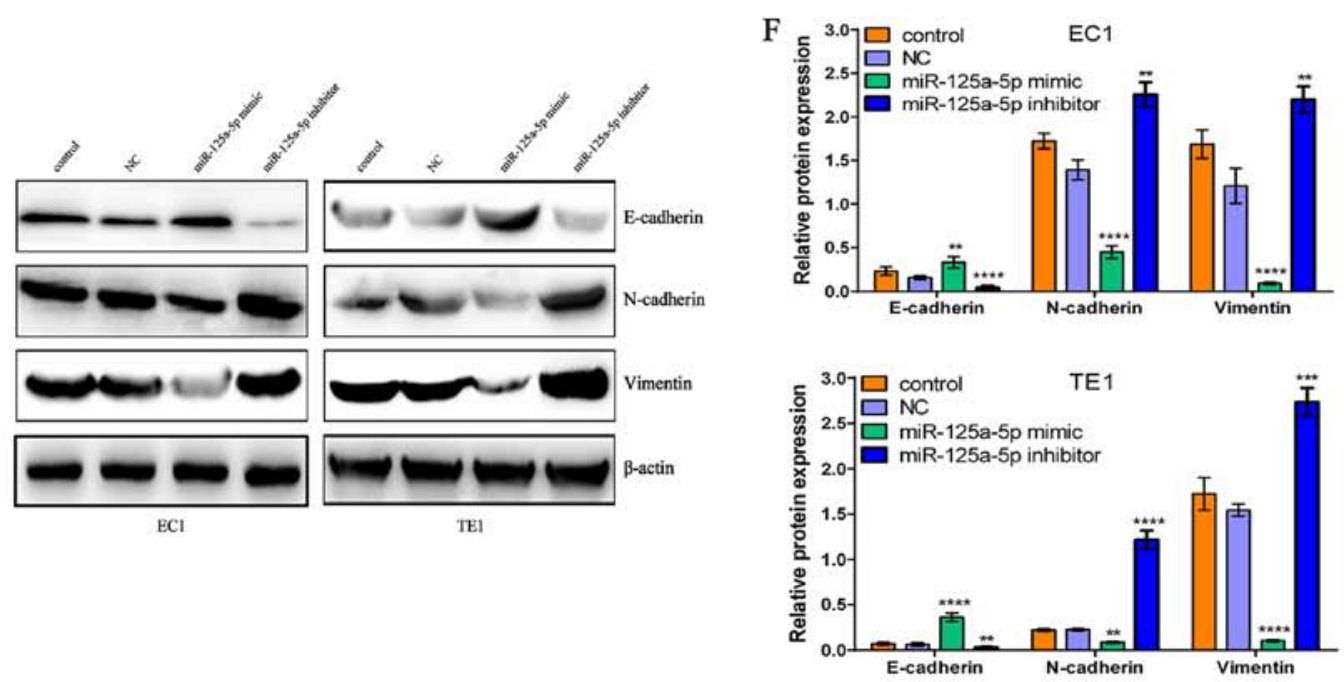

Figure 3. The miR-125a-5p-mediated changes in the migratory and invasive abilities of esophageal squamous cell carcinoma (ESCC) cells are tightly associated with the epithelial-mesenchymal transition (EMT) process. (A) miR-125a-5p overexpression or downregulation evidently reduced or promoted the migratory ability of EC1 and TE1 ESCC cells, respectively. (B) Migration distance was counted to evaluate the migratory ability of EC1 and TE1 cells; ${ }^{*} \mathrm{P}<0.05$ and ${ }^{* *} \mathrm{P}<0.01$, compared with the control group and NC group. (C) miR-125a-5p overexpression or downregulation markedly suppressed or enhanced the invasive ability of EC1 and TE1 ESCC cells. (D) Statistical analysis of the invasive numbers of EC1 and TE1 ESCC cells; "P<0.05 and ${ }^{* *} \mathrm{P}<0.01$, compared with the control group and NC group. (E) miR-125a-5p overexpression suppressed the protein expression levels of N-cadherin and Vimentin, and promoted E-cadherin protein expression; miR-125a-5p downregulation promoted the expression levels of N-cadherin and Vimentin proteins, and suppressed E-cadherin protein expression in EC1 and TE1 ESCC cells. Western blot analysis was utilized to investigate the expression levels of the EMT-related molecular markers, E-cadherin, $\mathrm{N}$-cadherin and Vimentin, and $\beta$-actin was used as a loading control. (F) Relative levels of E-cadherin, N-cadherin and Vimentin in ESCC EC1 and TE1 cells; ${ }^{* *} \mathrm{P}<0.01,{ }^{* * *} \mathrm{P}<0.001$ and ${ }^{* * * *} \mathrm{P}<0.0001$, compared with the control group and NC group. 
A
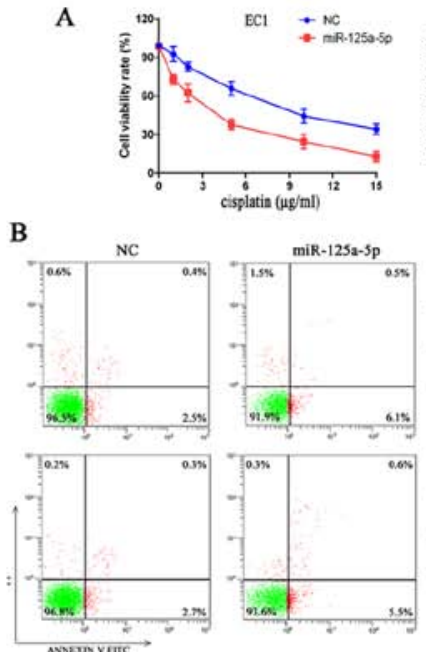

EC1

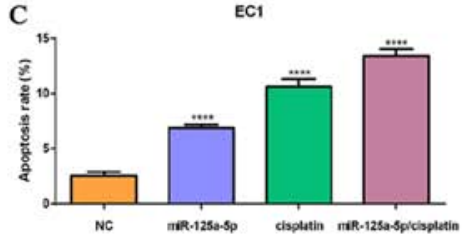

$\mathrm{F}$

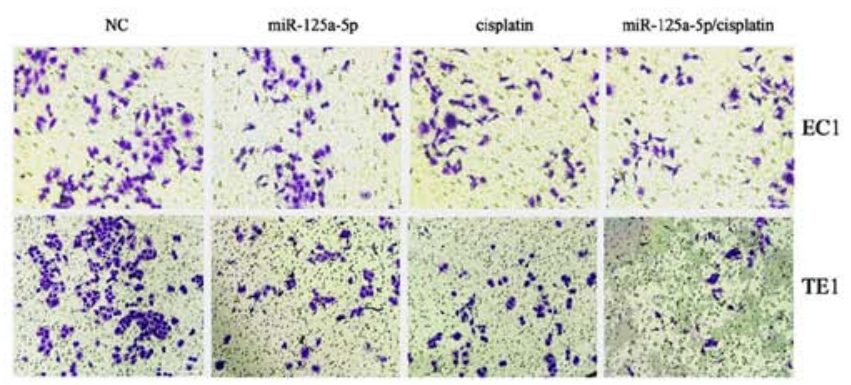

G

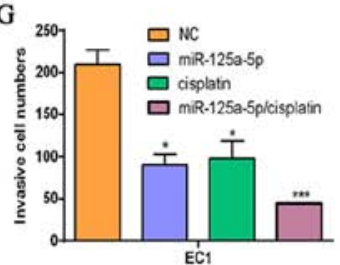

I

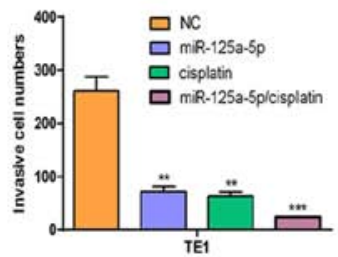

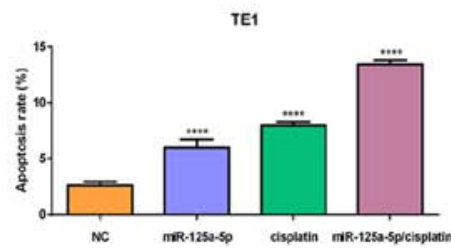
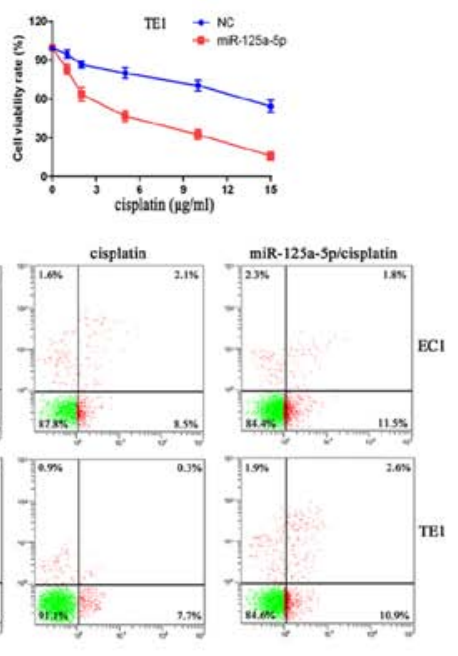

ECI
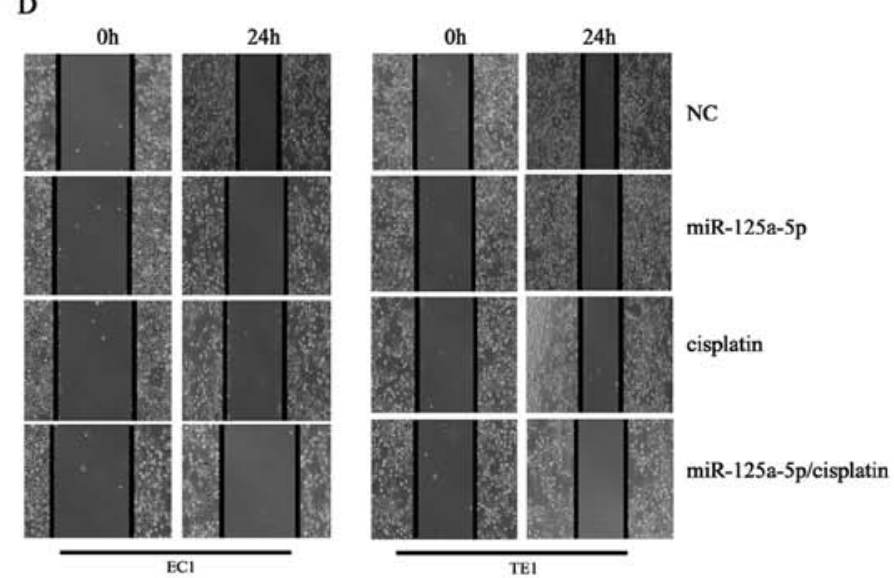

E

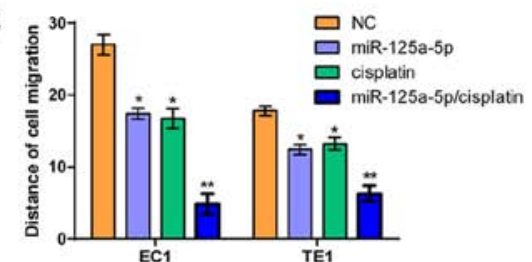

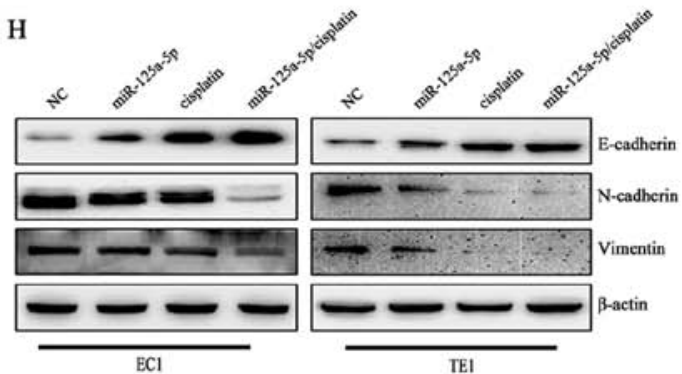
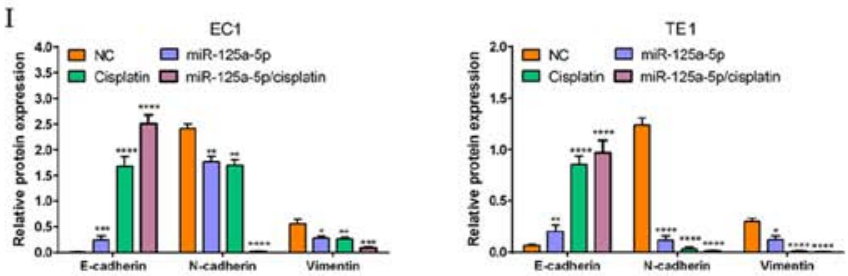

Figure 4. miR-125a-5p enhances the sensitivity of esophageal squamous cell carcinoma (ESCC) cells to cisplatin. (A) miR-125a-5p increased the cell-killing effects of cisplatin on EC1 and TE1 ESCC cells. (B) miR-125a-5p combined with cisplatin significantly promoted the apoptosis of EC1 and TE1 ESCC cells (C) Statistical analysis of the numbers of apoptotic EC1 and TE1 ESCC cells subjected to the different treatments; ${ }^{* * * * *} \mathrm{P}<0.0001$, compared with NC group. (D) miR-125a-5p in combination with cisplatin significantly inhibited the migratory ability of EC1 and TE1 ESCC cells. (E) Migration distance was counted to evaluate the migratory ability of EC1 and TE1 cells; ${ }^{*} \mathrm{P}<0.05$ and ${ }^{* *} \mathrm{P}<0.01$, compared with the NC group. (F) miR-125a-5p in combination with cisplatin significantly inhibited the invasive ability of EC1 and TE1 ESCC cells. (G) Statistical analysis of the invasive numbers of EC1 and TE1 ESCC cells subjected to various treatments; ${ }^{*} \mathrm{P}<0.05,{ }^{* *} \mathrm{P}<0.01$ and ${ }^{* * *} \mathrm{P}<0.001$, compared with the NC group. (H) miR-125a-5p in combination with cisplatin promoted E-cadherin expression, and suppressed the expression levels of N-cadherin and Vimentin in EC1 and TE1 ESCC cells. Western blot analysis was utilized to examine the expression levels of the EMT-related molecular markers, E-cadherin, N-cadherin and Vimentin, and $\beta$-actin was used as a loading control. (I) Relative levels of E-cadherin, $\mathrm{N}$-cadherin and Vimentin in EC1 and TE1 ESCC cells, ${ }^{*} \mathrm{P}<0.05,{ }^{* * *} \mathrm{P}<0.01,{ }^{* * * *} \mathrm{P}<0.001$ and ${ }^{* * * *} \mathrm{P}<0.0001$, compared with the NC group.

reported activator of the STAT3 signaling pathway) can abolish the inactivation of the STAT3 signaling pathway induced by miR-125a-5p in combination with cisplatin in ESCC cells, western blot analysis was employed to examine the activation status of the STAT3 signaling pathway in the EC1 and TE1 cells. The results indicated that IL- 6 markedly increased the protein expression levels of t-STAT3, p-STAT3 and VEGF, compared with the miR-125a-5p/cisplatin group $(\mathrm{P}<0.01)$ in the
EC1 and TE1 cells (Fig. 6C and D), suggesting that IL-6 can re-activate the STAT3 signaling pathway in ESCC cells treated with a combination of miR-125a-5p and cisplatin.

STAT3 activation evoked by IL-6 attenuates the suppressive effects mediated by miR-125a-5p/cisplatin on the proliferation and invasion of ESCC cells and also attenuates the pro-apoptotic effects. To further determine whether STAT3 activation 

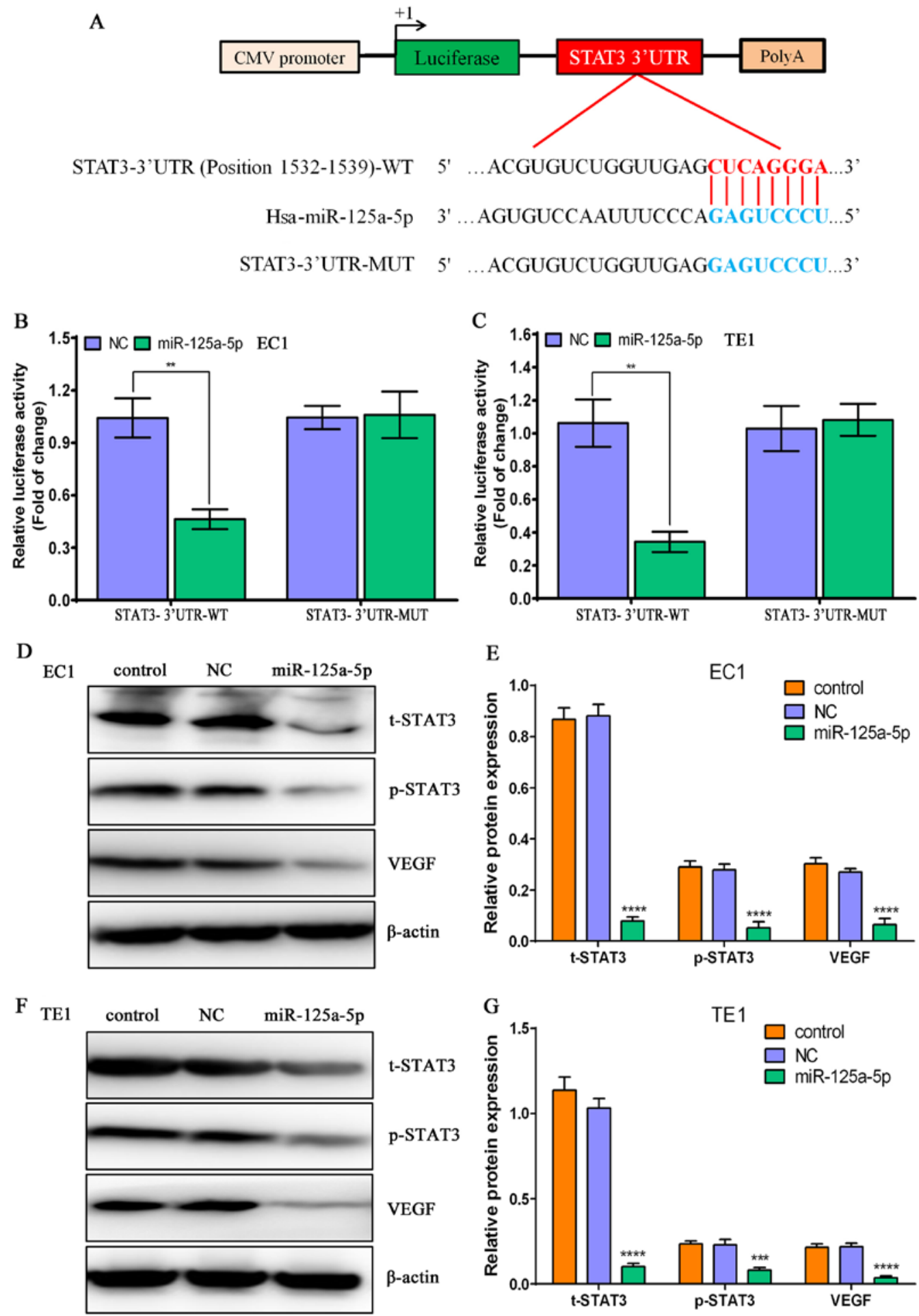

Figure 5. STAT3 is a direct target of miR-125a-5p in esophageal squamous cell carcinoma (ESCC) cells. (A) The signal transducer and activator of transcription-3 (STAT3) 3'-UTR sequences including wild-type (WT) or mutant (MUT) were inserted into the downstream of luciferase reporter vector according to diagrammatic presentation. (B) The luciferase activity was determined by co-transfecting the vectors (STAT3 3'-UTR-WT and MUT) combined with NC or miR-125a-5p mimic into EC1 ESCC cells; ${ }^{* *} \mathrm{P}<0.01$, compared with the NC group. (C) The luciferase activity was determined by co-transfecting the vectors (STAT3 3'-UTR-WT and MUT) combined with NC or miR-125a-5p mimic into TE1 ESCC cells; ${ }^{* *} \mathrm{P}<0.01$, compared with the NC group. (D) Western blot analysis of the protein expression levels of t-STAT3, p-STAT3 and vascular endothelial growth factor (VEGF) in the EC1 ESCC cells subjected to various treatments, and $\beta$-actin was used as a loading control. (E) Relative protein levels of t-STAT3, p-STAT3 and VEGF in EC1 ESCC cells subjected to different treatments; ${ }^{* * * * *} \mathrm{P}<0.0001$, compared with the control group and NC group. (F) Western blot analysis of the protein expression levels of t-STAT3, p-STAT3 and VEGF in TE1 ESCC cells subjected to various treatments, and $\beta$-actin was used as a loading control. (G) Relative protein levels of t-STAT3, p-STAT3 and VEGF in TE1 ESCC cells subjected to various treatments; ${ }^{* * * *} \mathrm{P}<0.001$ and ${ }^{* * * * * *} \mathrm{P}<0.0001$, compared with the control group and $\mathrm{NC}$ group.

can partially recover the phenotype of ESCC cells, which had been altered by miR-125a-5p/cisplatin, IL- 6 was used to treat the ESCC cells, and CCK-8, flow cytometry and Transwell chamber assay were used to examine the status of proliferation, apoptosis and invasion of EC1 and TE1 cells, respectively. We found that miR-125a-5p/cisplatin/IL-6 treatment significantly 
A
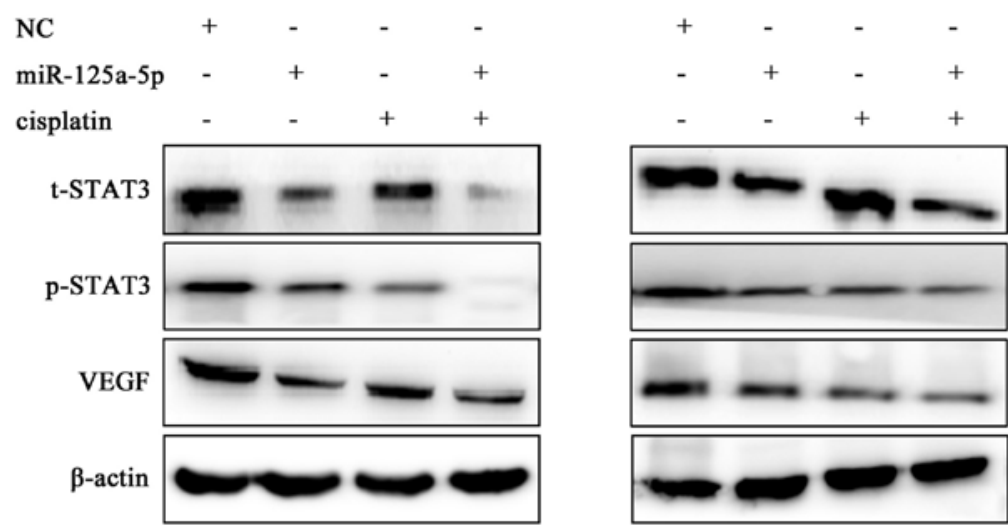

B

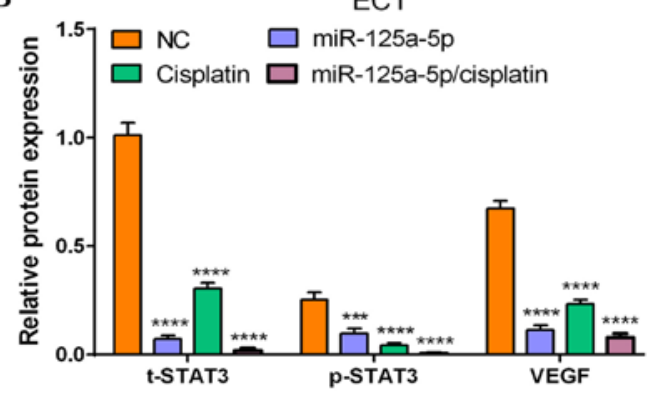

C

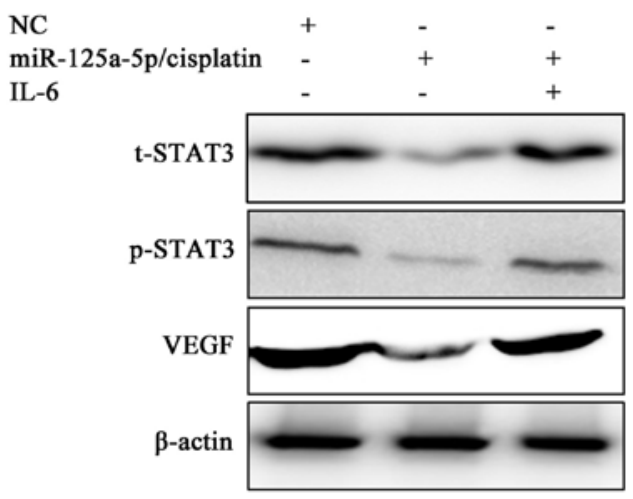

D

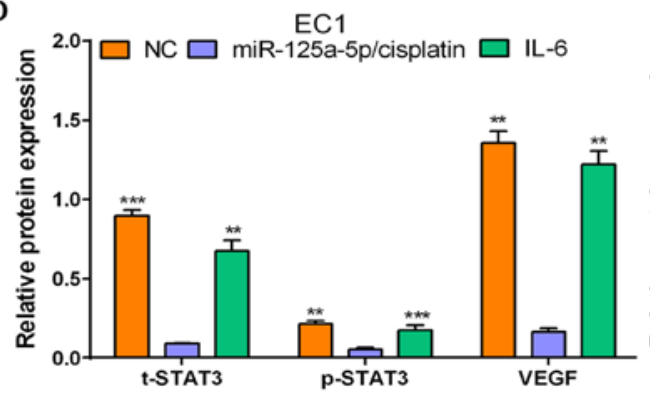

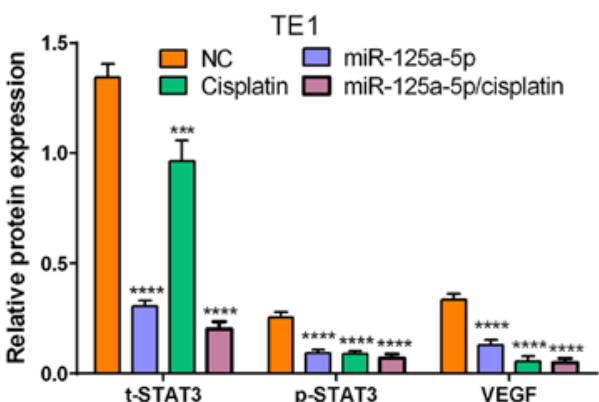

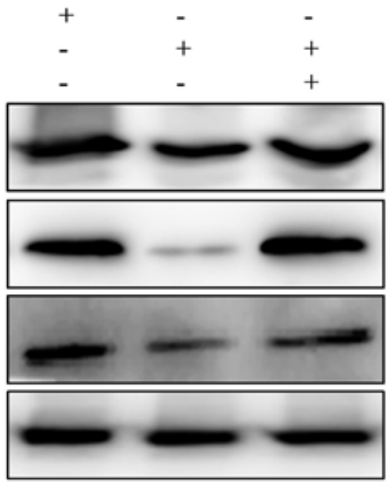

TE1

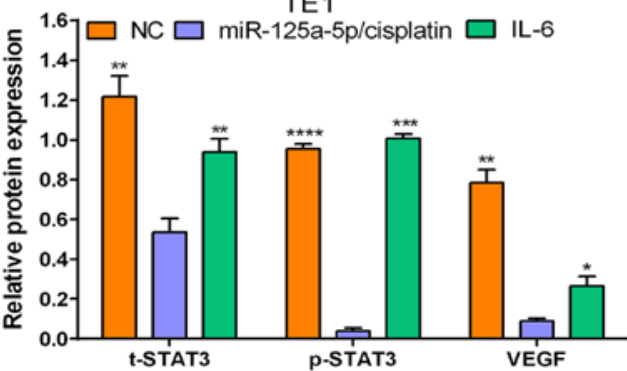

Figure 6. miR-125a-5p/cisplatin suppresses signal transducer and activator of transcription-3 (STAT3) activation and vascular endothelial growth factor (VEGF) expression, and the STAT3 activator, interleukin (IL)-6 re-activates the STAT3 signaling pathway in esophageal squamous cell carcinoma (ESCC) cells treated with miR-125a/cisplatin. (A) Western blot analysis of the protein expression levels of t-STAT3, p-STAT3 and VEGF in the NC group, miR-125a-5p alone group, cisplatin alone group and miR-125a-5p/cisplatin combination group, and $\beta$-actin was employed as a loading control. (B) The relative protein levels of t-STAT3, p-STAT3 and VEGF in the NC group, miR-125a-5p alone group, cisplatin alone group and miR-125a-5p/cisplatin combination group; ${ }^{* * *} \mathrm{P}<0.001$ and ${ }^{* * * *} \mathrm{P}<0.0001$, compared with the NC group. (C) Western blot analysis of the protein expression levels of t-STAT3, p-STAT3 and VEGF in the NC group, miR-125a-5p/cisplatin combination group and miR-125a-5p/cisplatin/IL-6 combination group, and $\beta$-actin was employed as a loading control. (D) The relative protein levels of t-STAT3, p-STAT3 and VEGF in the NC group, miR-125a-5p/cisplatin combination group and miR-125a-5p/cisplatin/IL-6 combination group; ${ }^{*} \mathrm{P}<0.05,{ }^{* *} \mathrm{P}<0.01,{ }^{* * *} \mathrm{P}<0.001$ and ${ }^{* * * * *} \mathrm{P}<0.0001$, compared with the miR-125a-5p/cisplatin combination group.

increased the viability of the EC1 and TE1 cells at 24, 48 and $72 \mathrm{~h}$, compared with the miR-125a-5p/cisplatin group (Fig. 7A).
Cell apoptosis assay revealed that miR-125a-5p/cisplatin/IL-6 treatment markedly decreased the apoptosis of the EC1 and 
A

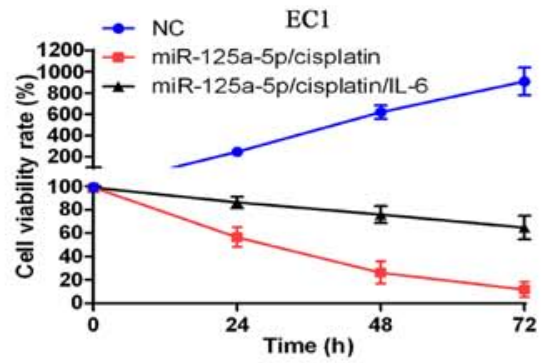

B
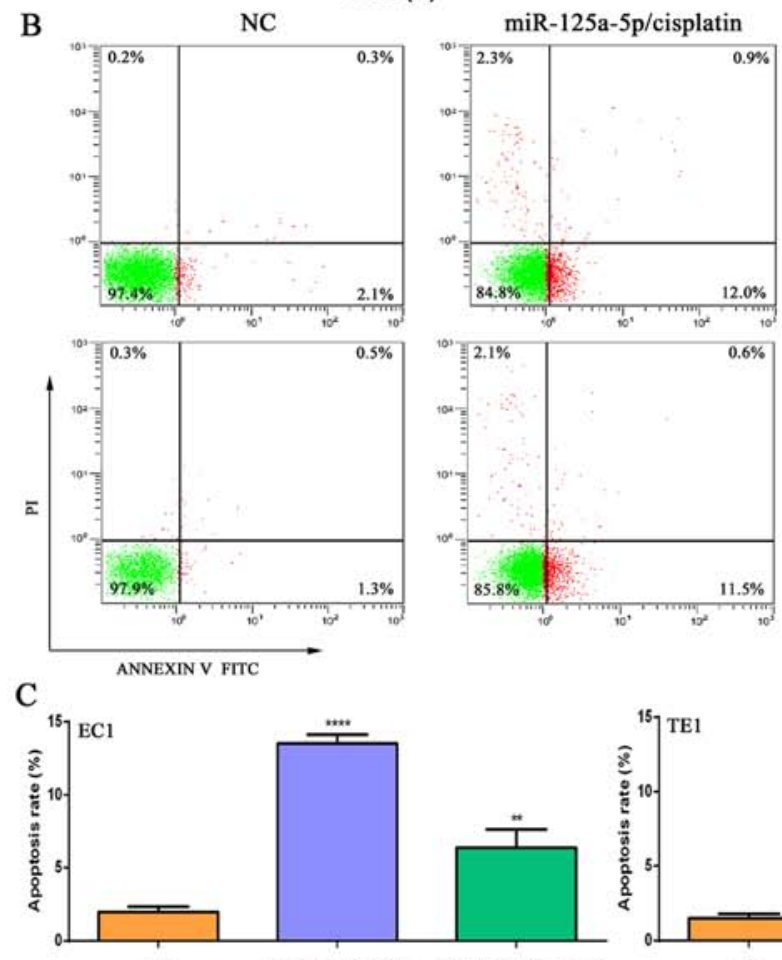

NC
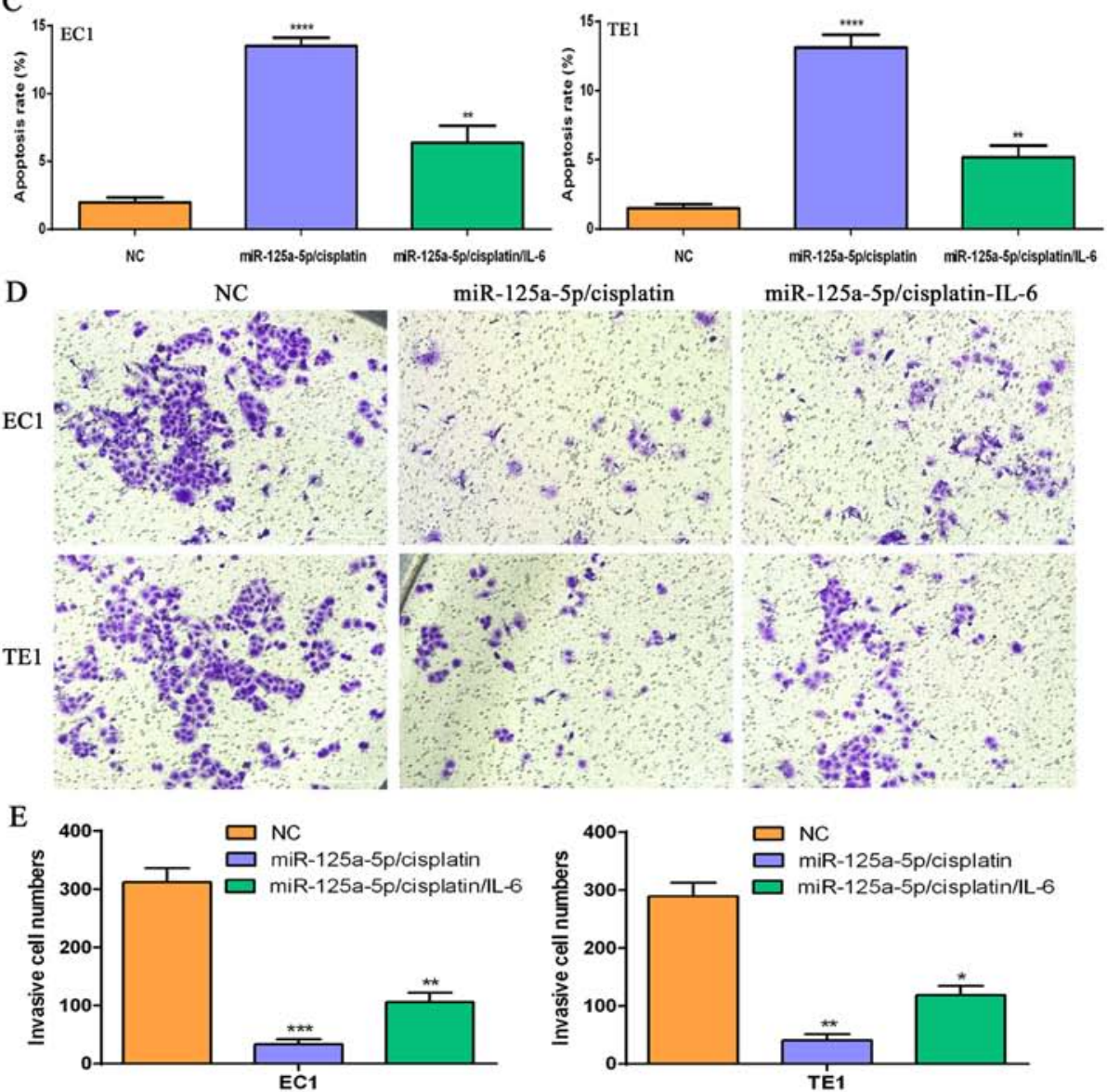

miR-125a-5p/cisplatin-IL-6

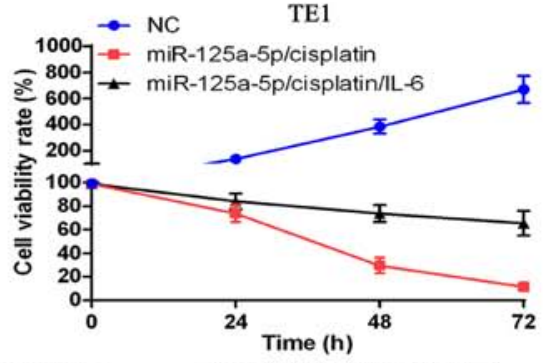

$\mathrm{miR}-125 \mathrm{a}-5 \mathrm{p} /$ cisplatin/IL-6
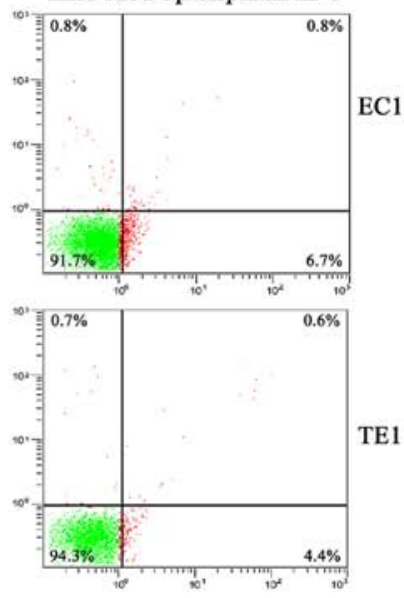

C1

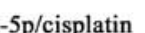

(1)
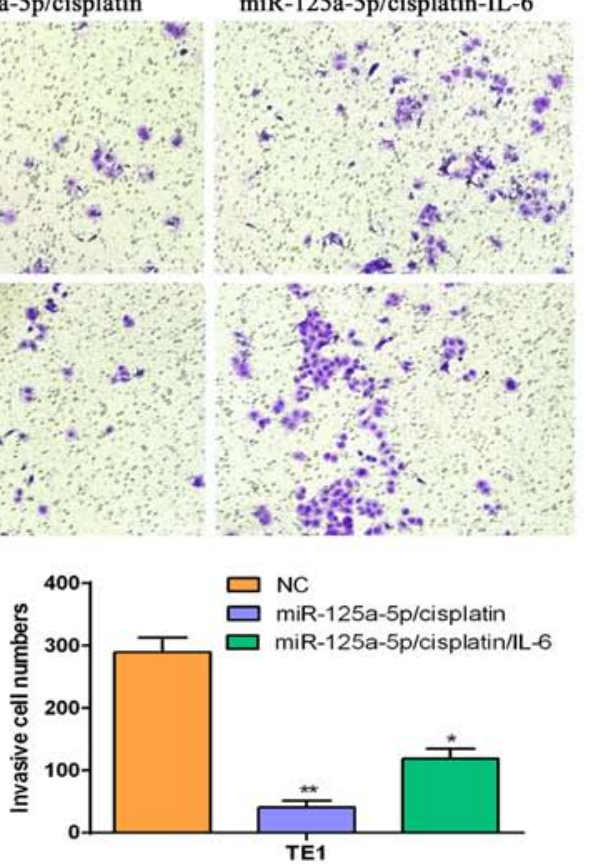

Figure 7. The signal transducer and activator of transcription-3 (STAT3) activator, interleukin (IL)-6 reverses the alteration in the cell phenotype mediated by miR-125a-5p/cisplatin in esophageal squamous cell carcinoma (ESCC) cells. (A) CCK-8 kit assay for cell proliferation in the NC group, miR-125a-5p/cisplatin combination group and miR-125a-5p/cisplatin/IL-6 combination group. (B) Flow cytometry of cell apoptosis in the NC group, miR-125a-5p/cisplatin combination group and miR-125a-5p/cisplatin/IL-6 combination group. (C) Statistical analysis of the apoptotic cell numbers in the NC group, miR-125a-5p/cisplatin combination group and miR-125a-5p/cisplatin/IL-6 combination group; ${ }^{* *} \mathrm{P}<0.01$ and ${ }^{* * * *} \mathrm{P}<0.0001$, compared with the NC group. (D) Transwell chamber assay for cell invasive ability in the $\mathrm{NC}$ group, miR-125a-5p/cisplatin combination group and miR-125a-5p/cisplatin/IL-6 combination group. (E) Statistical analysis of the invasive cell numbers in the NC group, miR-125a-5p/cisplatin combination group and miR-125a-5p/cisplatin/IL-6 combination group, ${ }^{*} \mathrm{P}<0.05,{ }^{* *} \mathrm{P}<0.01$ and ${ }^{* * *} \mathrm{P}<0.001$, compared with the NC group. 
TE1 cells, compared with the miR-125a-5p/cisplatin group $(\mathrm{P}<0.01)$ (Fig. 7B and $\mathrm{C})$. Notably, we found that miR-125a-5p/cisplatin/IL-6 treatment evidently restored the invasive ability of the EC1 and TE1 cells, compared with the miR-125a-5p/cisplatin group $(\mathrm{P}<0.05)$ (Fig. 7D and E). These data suggest that miR-125a-5p/cisplatin exerts antitumor effects by inhibiting the activation of the STAT3 signaling pathway.

\section{Discussion}

Mounting evidence has demonstrated that miRNAs function as either oncogenes or tumor suppressor genes in various type tumors, which may bring forth new challenges or may open up novel opportunities for the use of miRNAs as novel molecular targets for a myriad of tumors $(48,49)$. Moreover, miRNAs have been verified as a therapeutic tool in the management of pancreatic adenocarcinoma in clinical studies $(50,51)$, suggesting that miRNAs have important clinical value in many different types of tumors. Therefore, the identification of key miRNA molecules implicated in the development and progression of ESCC may provide new diagnostic and prognostic markers, and may aid in the development of more effective treatment strategies for patients with ESCC. In the current study, we found that miR-125a-5p was downregulated in ESCC tissues and cells, implying that miR-125a-5p may function as a tumor suppressor in ESCC. Further analysis revealed that a decreased miR-125a-5p expression was tightly associated with a higher tumor staging and a lower survival rate of patients with ESCC, suggesting that miR-125a-5p participates in tumor development and progression; thus, miR-125a-5p may be a molecular marker for the malignant degree and prognosis of patients with ESCC.

There is strong evidence that miRNAs are tightly implicated in a mass of complex regulatory networks of crucial genes in a variety of tumors (52-54). It has been indicated that target genes of miRNAs are direct regulators of the hallmarks of cancer, including proliferation, cell cycle and cell apoptosis (52). Tao et al found that a decreased miR-125a expression in osteosarcoma tissues, and its overexpression contributed to growth suppression in osteosarcoma cells by downregulating E2F2 expression (55). Moreover, miR-125a-5p overexpression has been shown to markedly inhibit cell proliferation and tumor formation in retinoblastoma, exerting antitumor effects by suppressing the transcriptional co-activator with PDZ binding motif (TAZ) (36), a critical downstream component of the Hippo signaling pathway; TAZ overexpression has been shown to markedly accelerate tumor initiation and progression (56,57). Qin et al confirmed that miR-125a-5p overexpression significantly suppressed the proliferation and migratory ability of cervical cancer cells through the direct targeted inhibition of ABL2 expression (37). Converse results from leukemia have revealed that miR-125a overexpression induces daunorubicin resistance by suppressing the apoptosis of HL-60, K562 and THP-1 cells, which was further verified to be directly correlated with the downregulation of GRK2 and Puma (58). These findings fully highlighted the essential role of miR-125a-5p in the regulation of proliferation and apoptosis in a plethora of tumors. In this study, we found that miR-125a-5p overexpression significantly inhibited the proliferation, arrested the cell cycle in the G0/G1 phase and induced the apoptosis of ESCC cells, whereas miR-125a-5p downregulation markedly promoted the proliferation and cell cycle progression, and inhibited the apoptosis of ESCC cells. These findings suggest that miR-125a-5p may be an important regulator of cell proliferation, cell cycle and apoptosis in ESCC, and thus the manipulation of miR-125a-5p may be a novel molecular target for ESCC.

Understanding the possible mechanisms of tumor invasion and metastasis remains a formidable challenge for a large number of tumors. Several studies have focused on the involvement of miRNAs in tumor invasion and metastasis (59-62). The EMT process has been reported to be involved in tumor progression and metastasis, which may be a pivotal mechanism of tumor invasion and metastasis $(63,64)$. Recently, increasing evidence has demonstrated that miRNAs implicated in tumor invasion and metastasis are tightly associated with the EMT process (65-68). To further interpret the possible role of miR-125a-5p in invasion and metastasis of ESCC, we further examined the alterations in the migratory and invasive abilities of ESCC cells triggered by miRNA-125a-5p. We found that miR-125a-5p overexpression significantly reduced the migratory and invasive abilities of ESCC cells, whereas its downregulation promoted the migratory and invasive abilities of ESCC cells. Further analysis revealed that transfection with miR-125a-5p mimic markedly upregulated the protein expression levels of $\mathrm{N}$-cadherin and Vimentin, and downregulated the E-cadherin protein level in ESCC, and converse results were observed in the miR-125a-5p inhibitor group, implying that the involvement of miR-125a-5p in tumor invasion and metastasis may be partly achieved through the modulation of EMT-related signaling pathways. These findings potentiate miR-125a-5p as a potential predictor for the invasion and metastasis of ESCC.

Chemoresistance is a major hurdle in the treatment of many tumor patients. Recent studies have revealed that aberrant miRNA levels are tightly implicated in chemoresistance or chemosensitivity in a host of tumors (69-74), suggesting that targeting miRNAs to eradicate chemoresistance or improve chemosensitivity may be a novel therapeutic strategy for the therapy of tumor patients. Nishida et al found that miR-125a-5p evidently suppressed cell proliferation via targeting ERBB2, and miR-125a-5p markedly enhanced the sensitivity of gastric carcinoma cells to trastuzumab (75). Another study revealed that miR-200a increased the chemoresistance of breast cancer cells to chemotherapeutic agents; by contrast, miR-200a downregulation promoted the sensitivity of resistant cancer cells to gemcitabine (70). Furthermore, the re-introduction of miR-31 has been shown to significantly promote clonogenic resistance to cisplatin and carboplatin in NCI-H2452 cells without miR-31 expression (76). Another study revealed that miR-125a overexpression enhanced the sensitivity of paclitaxel on paclitaxel-resistant colon cancer cells (38). These findings highlight the crucial role of miRNAs in potentiating chemosensitivity in the process of tumor therapy. However, whether miR-125a-5p is implicated in the chemosensitivity of ESCC cells remains unknown. In this study, as expected, miR-125a-5p significantly elevated the killing efficacy of cisplatin on ESCC cells, and miR-125a-5p/cisplatin significantly promoted the apoptosis and reduced the migratory and invasive abilities of ESCC cells. Further analysis revealed that co-treatment with miR-125a-5p 
and cisplatin markedly increased the E-cadherin level and reduced the levels of $\mathrm{N}$-cadherin and Vimentin, suggesting that the miR-125a-5p-mediated enhancement of the cisplatin sensitivity of ESCC cells may be tightly associated with the suppression of the EMT process. The data presented herein suggest that miR-125a-5p markedly improved the therapeutic efficacy of cisplatin in ESCC, and thus miR-125a-5p may be a novel ancillary drug of cisplatin for use in the treatment of patients with ESCC in the future.

Several studies have demonstrated that the STAT3 signaling pathway is implicated in drug resistance in a variety of tumors $(77,78)$, and its activation not only promotes tumor growth rapidly, but also imparts therapeutic resistance in cancer cells (77,79-81). In this study, we determined whether the involvement of miRNA-125a-5p in the sensitivity of ESCC cells to cisplatin may be tightly associated with the activated status of the STAT3 signaling pathway. Therefore, we performed a search for the potential target genes of miR-125a-5p using TargetScan, miRanda and miRDB, and found that STAT3 was the direct target gene of miR-125a-5p by luciferase reporter assay. Further analysis demonstrated that miR-125a-5p overexpression significantly reduced the protein levels of t-STAT3, p-STAT3 and its downstream target gene, VEGF, ESCC cells. These findings suggest that the miR-125a-5p-mediated chemosensitivity may be tightly associated with the inactivation of the STAT3 signaling pathway in ESCC. Correspondingly, whether the re-activation of the STAT3 signaling pathway can reverse the chemosensitivity mediated by miR-125a-5p overexpression in ESCC, was examined. IL-6 [an activator of the STAT3 signaling pathway (39)] was used to re-activate the the STAT3 signaling pathway in ESCC cells. We found that IL-6 significantly recovered the t-STAT3 and p-STAT3 levels which were suppressed by miR-125a-5p/cisplatin in the ESCC cells, and further analysis revealed that the re-activation of the STAT3 signaling pathway evoked by IL- 6 significantly recovered cell viability, decreased cell apoptosis and promoted the invasion of ECSS cells co-treated with miR-125a-5p and cisplatin. The data presented herein suggest that miR-125a-5p enhances the sensitivity of ESCC cells to cisplatin via suppressing the activation of the STAT3 signaling pathway, which may be an underlying molecular mechanism through which miR-125a-5p exerts antitumor effects on ESCC.

In conclusion, the data from the present study suggest that a low level of miR-125a-5p is tightly associated with a higher tumor staging and a poor prognosis of patients with ESCC. The overexpression of miR-125a-5p significantly suppressed the proliferation, arrested the cell cycle in G0/G1 phase, induced the apoptosis and reduced the migratory and invasive abilities of ESCC cells, and converse results were observed in miR-125a-5p inhibitor group, which may be closely associated with the EMT process. Further analysis indicated that miR-125a-5p enhanced the sensitivity of ESCC cells to cisplatin, which may be achieved by the inactivation of the STAT3 signaling pathway. Most importantly, the re-activation of the STAT3 signaling pathway triggered by IL-6 prominently led to a recovery of the viability, decreased cell apoptosis and an increased cell invasive ability of ESCC cells. The data presented herein may provide a novel therapeutic strategy for the therapy of patients with ESCC by the combined use of miR-125a-5p with cisplatin.

\section{Acknowledgements}

Not applicable.

\section{Funding}

The present study was supported by the National Natural Science Foundation of China (no. 81272691) and the Key Scientific Research Projects of Henan Higher Education Institutions (no. 17A180016).

\section{Availability of data and materials}

All data generated or analyzed during this study are included in this published article.

\section{Authors' contributions}

QF supervised the whole project. QF, YZ and KM designed the study. YZ, KM and SY performed the majority of the experiments; XZ contributed to plasmid construction and luciferase reporter assay; FW performed the CCK-8 experiment; XZ and HL participated in the design and interpretation of some of the experiments; YZ and QF interpreted all the results and wrote the manuscript.

\section{Ethics approval and consent to participate}

This study was approved by the Institutional Research Ethics Committee of Zhengzhou University. All samples were obtained with informal written and none of the patients had received any treatments prior to surgery.

\section{Consent for publication}

Not applicable.

\section{Competing interests}

The authors declare that they have no competing interests.

\section{References}

1. Torre LA, Bray F, Siegel RL, Ferlay J, Lortet-Tieulent J and Jemal A: Global cancer statistics, 2012. CA Cancer J Clin 65: 87-108, 2015.

2. Conteduca V, Sansonno D, Ingravallo G, Marangi S, Russi S, Lauletta $\mathrm{G}$ and Dammacco F: Barrett's esophagus and esophageal cancer: An overview. Int J Oncol 41: 414-424, 2012.

3. Arnold M, Soerjomataram I, Ferlay J and Forman D: Global incidence of oesophageal cancer by histological subtype in 2012 . Gut 64: 381-387, 2015.

4. Berger AC, Farma J, Scott WJ, Freedman G, Weiner L, Cheng JD, Wang $\mathrm{H}$ and Goldberg $\mathrm{M}$ : Complete response to neoadjuvant chemoradiotherapy in esophageal carcinoma is associated with significantly improved survival. J Clin Oncol 23: 4330-4337, 2005.

5. Jemal A, Siegel R, Xu J and Ward E: Cancer statistics, 2010. CA Cancer J Clin 60: 277-300, 2010.

6. Law S, Kwong DL, Kwok KF, Wong KH, Chu KM, Sham JS and Wong J: Improvement in treatment results and long-term survival of patients with esophageal cancer: Impact of chemoradiation and change in treatment strategy. Ann Surg 238: 339-3488, 2003.

7. Sjoquist KM, Burmeister BH, Smithers BM, Zalcberg JR, Simes RJ, Barbour A and Gebski V; Australasian Gastro-Intestinal Trials Group: Survival after neoadjuvant chemotherapy or chemoradiotherapy for resectable oesophageal carcinoma: An updated meta-analysis. Lancet Oncol 12: 681-692, 2011. 
8. Pennathur A, Gibson MK, Jobe BA and Luketich JD: Oesophageal carcinoma. Lancet 381: 400-412, 2013.

9. Hong L, Han Y, Zhang H and Fan D: Prognostic markers in esophageal cancer: From basic research to clinical use. Expert Rev Gastroenterol Hepatol 9: 887-889, 2015.

10. Haenisch S and Cascorbi I: miRNAs as mediators of drug resistance. Epigenomics 4: 369-381, 2012.

11. Ambros V: The functions of animal microRNAs. Nature 431: 350-355, 2004

12. Bartel DP: MicroRNAs: Genomics, biogenesis, mechanism, and function. Cell 116: 281-297, 2004.

13. Gurtan AM and Sharp PA: The role of miRNAs in regulating gene expression networks. J Mol Biol 425: 3582-3600, 2013.

14. Ameres SL and Zamore PD: Diversifying microRNA sequence and function. Nat Rev Mol Cell Biol 14: 475-488, 2013.

15. Mendell JT: MicroRNAs: Critical regulators of development, cellular physiology and malignancy. Cell Cycle 4: 1179-1184 2005.

16. Esteller M: Non-coding RNAs in human disease. Nat Rev Genet 12: 861-874, 2011.

17. Tiscornia G and Izpisúa Belmonte JC: MicroRNAs in embryonic stem cell function and fate. Genes Dev 24: 2732-2741, 2010.

18. Inui M, Martello G and Piccolo S: MicroRNA control of signal transduction. Nat Rev Mol Cell Biol 11: 252-263, 2010.

19. Ping W, Gao Y,Fan X, Li W, Deng Y and Fu X: MiR-181a contributes gefitinib resistance in non-small cell lung cancer cells by targeting GAS7. Biochem Biophys Res Commun 495: 2482-2489, 2018.

20. Gartel AL and Kandel ES: miRNAs: Little known mediators of oncogenesis. Semin Cancer Biol 18: 103-110, 2008.

21. Iorio MV and Croce CM: microRNA involvement in human cancer. Carcinogenesis 33: 1126-1133, 2012.

22. Negrini M, Nicoloso MS and Calin GA: MicroRNAs and cancer-new paradigms in molecular oncology. Curr Opin Cell Biol 21: 470-479, 2009

23. Spizzo R, Nicoloso MS, Croce CM and Calin GA: SnapShot: microRNAs in cancer. Cell 137: 586-586.e581, 2009.

24. Voorhoeve PM: MicroRNAs: Oncogenes, tumor suppressors or master regulators of cancer heterogeneity? Biochim Biophys Acta 1805: 72-86, 2010

25. Calin GA and Croce CM: MicroRNA signatures in human cancers. Nat Rev Cancer 6: 857-866, 2006.

26. Esquela-Kerscher A and Slack FJ: Oncomirs - microRNAs with a role in cancer. Nat Rev Cancer 6: 259-269, 2006.

27. Hammond SM: MicroRNAs as tumor suppressors. Nat Genet 39: 582-583, 2007

28. Baraniskin A, Chomiak M, Ahle G, Gress T, Buchholz M, Turewicz M, Eisenacher M, Margold M, Schlegel U, Schmiegel W, et al: MicroRNA-30c as a novel diagnostic biomarker for primary and secondary B-cell lymphoma of the CNS. J Neurooncol 137: $463-468,2018$

29. Li C, Zheng X, Li W, Bai F, Lyu J and Meng QH: Serum miR-486-5p as a diagnostic marker in cervical cancer: With investigation of potential mechanisms. BMC Cancer 18: 61, 2018.

30. Yao XD, Li P and Wang JS: MicroRNA differential expression spectrum and microRNA-125a-5p inhibition of laryngeal cancer cell proliferation. Exp Ther Med 14: 1699-1705, 2017.

31. Li G, Zhang W, Gong L and Huang X: MicroRNA-125a-5p inhibits cell proliferation and induces apoptosis in hepatitis B virus-related hepatocellular carcinoma by downregulation of ErbB3. Oncol Res, 2017.

32. Coppola N, de Stefano G, Panella M, Onorato L, Iodice V, Minichini C, Mosca N, Desiato L, Farella N, Starace M, et al: Lowered expression of microRNA-125a-5p in human hepatocellular carcinoma and up-regulation of its oncogenic targets sirtuin-7, matrix metalloproteinase-11, and c-Raf. Oncotarget 8: 25289-25299, 2017.

33. Potenza N, Mosca N, Zappavigna S, Castiello F, Panella M, Ferri C, Vanacore D, Giordano A, Stiuso P, Caraglia M, et al: MicroRNA-125a-5p is a downstream effector of sorafenib in its antiproliferative activity toward human hepatocellular carcinoma cells. J Cell Physiol 232: 1907-1913, 2017.

34. Zhong L, Sun S, Shi J, Cao F, Han X and Chen Z: MicroRNA$125 a-5 p$ plays a role as a tumor suppressor in lung carcinoma cells by directly targeting STAT3. Tumour Biol 39: 1010428317697579, 2017.

35. Fu Y and Cao F: MicroRNA-125a-5p regulates cancer cell proliferation and migration through NAIF1 in prostate carcinoma. OncoTargets Ther 8: 3827-3835, 2015.
36. Zhang Y, Xue C, Zhu X, Zhu X, Xian $\mathrm{H}$ and Huang Z: Suppression of microRNA-125a-5p upregulates the TAZ-EGFR signaling pathway and promotes retinoblastoma proliferation. Cell Signal 28: 850-860, 2016.

37. Qin X, Wan Y, Wang S and Xue M: MicroRNA-125a-5p modulates human cervical carcinoma proliferation and migration by targeting ABL2. Drug Des Devel Ther 10: 71-79, 2015.

38. Chen J, Chen Y and Chen Z: MiR-125a/b regulates the activation of cancer stem cells in paclitaxel-resistant colon cancer. Cancer Invest 31: 17-23, 2013.

39. Kishimoto T: IL-6: From its discovery to clinical applications. Int Immunol 22: 347-352, 2010.

40. Lu Z, Liu H, Xue L, Xu P, Gong T and Hou G: An activated Notch1 signaling pathway inhibits cell proliferation and induces apoptosis in human esophageal squamous cell carcinoma cell line EC9706. Int J Oncol 32: 643-651, 2008.

41. Zafar S, Coates DE, Cullinan MP, Drummond BK, Milne T and Seymour GJ: Effects of zoledronic acid and geranylgeraniol on the cellular behaviour and gene expression of primary human alveolar osteoblasts. Clin Oral Investig 20: 2023-2035, 2016.

42. Nana-Sinkam SP and Croce CM: MicroRNA regulation of tumorigenesis, cancer progression and interpatient heterogeneity: Towards clinical use. Genome Biol 15: 445, 2014

43. Wang X, Ivan M and Hawkins SM: The role of MicroRNA molecules and MicroRNA-regulating machinery in the pathogenesis and progression of epithelial ovarian cancer. Gynecol Oncol 147: 481-487, 2017.

44. Kim J, Yao F, Xiao Z, Sun Y and Ma L: MicroRNAs and metastasis: Small RNAs play big roles. Cancer Metastasis Rev 37: 5-15, 2018.

45. Pastorkova Z, Skarda J and Andel J: The role of microRNA in metastatic processes of non-small cell lung carcinoma. Biomed Pap Med Fac Univ Palacky Olomouc Czech Repub 160: 343-357, 2016.

46. Mittal V: Epithelial mesenchymal transition in tumor metastasis. Annu Rev Pathol 13: 395-412, 2018.

47. Giannelli G, Koudelkova P, Dituri F and Mikulits W: Role of epithelial to mesenchymal transition in hepatocellular carcinoma. J Hepatol 65: 798-808, 2016.

48. Dorrance AM, Neviani P, Ferenchak GJ, Huang X, Nicolet D, Maharry KS, Ozer HG, Hoellarbauer P, Khalife J, Hill EB, et al: Targeting leukemia stem cells in vivo with antagomiR-126 nanoparticles in acute myeloid leukemia. Leukemia 29: 2143-2153, 2015.

49. Jiang X, Bugno J, Hu C, Yang Y, Herold T, Qi J, Chen P, Gurbuxani S, Arnovitz S, Strong J, et al: Eradication of acute myeloid leukemia with FLT3 ligand-targeted miR-150 nanoparticles. Cancer Res 76: 4470-4480, 2016.

50. Keklikoglou I, Hosaka K, Bender C, Bott A, Koerner C, Mitra D, Will R, Woerner A, Muenstermann E, Wilhelm H, et al: MicroRNA-206 functions as a pleiotropic modulator of cell proliferation, invasion and lymphangiogenesis in pancreatic adenocarcinoma by targeting ANXA 2 and KRAS genes. Oncogene 34: 4867-4878, 2015.

51. Papaconstantinou IG, Lykoudis PM, Gazouli M, Manta A, Polymeneas G and Voros D: A review on the role of microRNA in biology, diagnosis, and treatment of pancreatic adenocarcinoma. Pancreas 41: 671-677, 2012.

52. Robb T, Reid G and Blenkiron C: Exploiting microRNAs as cancer therapeutics. Target Oncol 12: 163-178, 2017.

53. Li Z, Peng Z, Gu S, Zheng J, Feng D, Qin Q and He J: Global analysis of miRNA-mRNA interaction network in breast cancer with brain metastasis. Anticancer Res 37: 4455-4468, 2017.

54. Cora' D, Re A, Caselle M and Bussolino F: MicroRNA-mediated regulatory circuits: Outlook and perspectives. Phys Biol 14: 045001, 2017.

55. Tao T, Shen Q, Luo J, Xu Y and Liang W: MicroRNA-125a regulates cell proliferation via directly targeting E2F2 in osteosarcoma. Cell Physiol Biochem 43: 768-774, 2017.

56. Hsu YL, Hung JY, Chou SH, Huang MS, Tsai MJ, Lin YS, Chiang SY, Ho YW, Wu CY and Kuo PL: Angiomotin decreases lung cancer progression by sequestering oncogenic YAP/TAZ and decreasing Cyr61 expression. Oncogene 34: 4056-4068, 2015.

57. Brusgard JL, Choe M, Chumsri S, Renoud K, MacKerell AD Jr, Sudol M and Passaniti A: RUNX2 and TAZ-dependent signaling pathways regulate soluble E-Cadherin levels and tumorsphere formation in breast cancer cells. Oncotarget 6: 28132-28150,2015.

58. Bai H, Zhou L, Wang C, Xu X, Jiang J, Qin Y, Wang X, Zhao C and Shao S: Involvement of miR-125a in resistance to daunorubicin by inhibiting apoptosis in leukemia cell lines. Tumour Biol 39: 1010428317695964, 2017. 
59. Palma Flores C, García-Vázquez R, Gallardo Rincón D, Ruiz-García E, Astudillo de la Vega H, Marchat LA, Salinas Vera YM and López-Camarillo C: MicroRNAs driving invasion and metastasis in ovarian cancer: Opportunities for translational medicine (Review). Int J Oncol 50: 1461-1476, 2017.

60. Verma V and Lautenschlaeger T: MicroRNAs in non-small cell lung cancer invasion and metastasis: From the perspective of the radiation oncologist. Expert Rev Anticancer Ther 16: 767-774, 2016.

61. Chan SH and Wang LH: Regulation of cancer metastasis by microRNAs. J Biomed Sci 22: 9, 2015.

62. Zhao X, Li X and Yuan H: microRNAs in gastric cancer invasion and metastasis. Front Biosci 18: 803-810, 2013.

63. Yang J and Weinberg RA: Epithelial-mesenchymal transition: At the crossroads of development and tumor metastasis. Dev Cell 14: 818-829, 2008.

64. Kalluri R and Weinberg RA: The basics of epithelial-mesenchymal transition. J Clin Invest 119: 1420-1428, 2009.

65. Chen W, Kong KK, Xu XK, Chen C, Li H, Wang FY, Peng XF, Zhang Z, Li P, Li JL, et al: Downregulation of miR-205 is associated with glioblastoma cell migration, invasion, and the epithelial-mesenchymal transition, by targeting ZEB1 via the Akt/mTOR signaling pathway. Int J Oncol 52: 485-495, 2018

66. Chen C, Yang Q, Wang D, Luo F, Liu X, Xue J, Yang P, Xu H, Lu J, Zhang A, et al: MicroRNA-191, regulated by HIF-2 $\alpha$, is involved in EMT and acquisition of a stem cell-like phenotype in arsenite-transformed human liver epithelial cells. Toxicol In Vitro 48: 128-136, 2018.

67. Huang J, He Y, Mcleod HL, Xie Y, Xiao D, Hu H, Chen P, Shen L, Zeng S, Yin X, et al: miR-302b inhibits tumorigenesis by targeting EphA2 via Wnt/ $\beta$-catenin/EMT signaling cascade in gastric cancer. BMC Cancer 17: 886, 2017.

68. Xu X, Cao L, Zhang Y, Lian H, Sun Z and Cui Y: MicroRNA-1246 inhibits cell invasion and epithelial mesenchymal transition process by targeting CXCR4 in lung cancer cells. Cancer Biomark 21: 251-260,2018.

69. Berman M, Mattheolabakis G, Suresh M and Amiji M: Reversing epigenetic mechanisms of drug resistance in solid tumors using targeted microRNA delivery. Expert Opin Drug Deliv 13: 987-998, 2016.

70. Yu SJ, Yang L, Hong Q, Kuang XY, Di GH and Shao ZM: MicroRNA-200a confers chemoresistance by antagonizing TP53INP1 and YAP1 in human breast cancer. BMC Cancer 18 $74,2018$.

71. Xiong J, Wang D, Wei A, Ke N, Wang Y, Tang J, He S, Hu W and Liu X: MicroRNA-410-3p attenuates gemcitabine resistance in pancreatic ductal adenocarcinoma by inhibiting HMGB1-mediated autophagy. Oncotarget 8: 107500-107512, 2017.

72. Leivonen SK, Icay K, Jäntti K, Siren I, Liu C, Alkodsi A, Cervera A, Ludvigsen M, Hamilton-Dutoit SJ, d'Amore F, et al: MicroRNAs regulate key cell survival pathways and mediate chemosensitivity during progression of diffuse large B-cell lymphoma. Blood Cancer J 7: 654, 2017.
73. Gabra MM and Salmena L: microRNAs and acute myeloid leukemia chemoresistance: A mechanistic overview. Front Oncol 7: 255, 2017

74. Yang RM, Zhan M, Xu SW, Long MM, Yang LH, Chen W, Huang S, Liu Q, Zhou J, Zhu J, et al: miR-3656 expression enhances the chemosensitivity of pancreatic cancer to gemcitabine through modulation of the RHOF/EMT axis. Cell Death Dis 8: e3129, 2017

75. Nishida N, Mimori K, Fabbri M, Yokobori T, Sudo T, Tanaka F Shibata K, Ishii H, Doki Y and Mori M: MicroRNA-125a-5p is an independent prognostic factor in gastric cancer and inhibits the proliferation of human gastric cancer cells in combination with trastuzumab. Clin Cancer Res 17: 2725-2733, 2011.

76. Moody HL, Lind MJ and Maher SG: MicroRNA-31 regulates chemosensitivity in malignant pleural mesothelioma. Mol Ther Nucleic Acids 8: 317-329, 2017.

77. Ara T, Nakata R, Sheard MA, Shimada H, Buettner R, Groshen SG, Ji L, Yu H, Jove R, Seeger RC, et al: Critical role of STAT3 in IL-6-mediated drug resistance in human neuroblastoma. Cancer Res 73: 3852-3864, 2013.

78. Suh YA, Jo SY, Lee HY and Lee C: Inhibition of IL-6/STAT3 axis and targeting Axl and Tyro3 receptor tyrosine kinases by apigenin circumvent taxol resistance in ovarian cancer cells. Int J Oncol 46: 1405-1411, 2015.

79. Garcia R, Bowman TL, Niu G, Yu H, Minton S, Muro-Cacho CA, Cox CE, Falcone R, Fairclough R, Parsons S, et al: Constitutive activation of Stat 3 by the Src and JAK tyrosine kinases participates in growth regulation of human breast carcinoma cells. Oncogene 20: 2499-2513, 2001

80. Epling-Burnette PK, Liu JH, Catlett-Falcone R, Turkson J, Oshiro M, Kothapalli R, Li Y, Wang JM, Yang-Yen HF, Karras J, et al: Inhibition of STAT3 signaling leads to apoptosis of leukemic large granular lymphocytes and decreased Mcl-1 expression. J Clin Invest 107: 351-362, 2001.

81. Huang W, Dong Z, Chen Y, Wang F, Wang CJ, Peng H, He Y, Hangoc G, Pollok K, Sandusky G, et al: Small-molecule inhibitors targeting the DNA-binding domain of STAT3 suppress tumor growth, metastasis and STAT3 target gene expression in vivo. Oncogene 35: 802, 2016.

This work is licensed under a Creative Common Attribution-NonCommercial-NoDerivatives 4.0 International (CC BY-NC-ND 4.0) License. 